\title{
Receptor Encoding of Moving Tactile Stimuli in Humans. I. Temporal Pattern of Discharge of Individual Low-Threshold Mechanoreceptors
}

\author{
Benoni B. Edin, ${ }^{1}$ Gregory K. Essick, ${ }^{2}$ Mats Trulsson, ${ }^{1}$ and Kurt $\AA$. Olsson ${ }^{1}$ \\ ${ }^{1}$ Department of Physiology, Umeå University, S-901 87 Umeå, Sweden and ${ }^{2}$ Dental Research Center and Curriculum in \\ Neurobiology, University of North Carolina at Chapel Hill, Chapel Hill, North Carolina 27599-7455
}

The response of 70 cutaneous, low-threshold mechanoreceptors in the human median, radial and inferior alveolar nerves to well controlled brush stimuli moving across the receptive field was quantitatively studied. Microneurography was used to obtain the response of each to multiple velocities from 0.5 to $32 \mathrm{~cm} / \mathrm{sec}$ in at least two opposing directions.

A high degree of response consistency was observed from the slowly adapting receptors to replication of the same stimulus and to a lesser, but significant degree from the fast adapting receptors. The evoked discharge reflected up to three partially overlapping phases of the moving stimulus: skin compression, indentation, and stretch. Although the overall discharge rate increased with both stimulus velocity and force, the spatial discharge pattern was preserved to a high degree. In contrast, the discharge patterns differed for opposing and orthogonal directions. Reducing the area of skin surrounding the receptive field that was contacted by the moving stimuli had little effect on the evoked response.

Individual mechanoreceptors display highly reliable differences to brush stimuli moving at different velocities. Moreover, different directions of movement evoke differences in the discharge that are consistently observed upon replication of the same stimuli. Despite the richness and consistency in the spatial discharge pattern displayed by individual receptors, it is argued that the details of the patterns are not likely used by the CNS to infer information about direction and velocity of movement across the skin. Rather, the intensity of discharge is proposed as a plausible information-bearing attribute of the stimulus-evoked response.

[Key words: human, microneurography, moving tactile stimuli, skin stretch, tactile motion, encoding, spatiotemporal patterns, mechanoreceptors, skin afferents, cutaneous mechanoreceptors, glabrous skin mechanoreceptors, hairy skin receptors, facial mechanoreceptors]

\footnotetext{
Received Apr. 5, 1994; revised July 11, 1994; accepted July 15, 1994.

The neurophysiological experiments were carried out at the Department of Physiology, Umea University, Sweden. The study was supported by the Swedish Medical Research Council (Grants 08667 and 00045), the Office of Naval Research, Arlington, VA (Grant 00014-90-J-1838), the National Institute of Dental Research (Program Project DE07509), the University of Umea, and the University of North Carolina School of Dentistry. We thank Mr. Göran Sandström and Mr. Anders Bäckström for technical support and Dr. Gary Koch, Professor of Biostatistics at the University of North Carolina, for statistical advice

Correspondence should be addressed to Benoni B. Edin, M.D., Ph.D., at the above address.
}

Copyright (C) 1995 Society for Neuroscience $0270-6474 / 95 / 150830-18 \$ 05.00 / 0$
Moving tactile stimuli have been extensively employed in the work of somatosensory psychologists and neurophysiologists since the 1800 s (e.g., see Hall and Donaldson, 1885). That moving tactile stimuli should prove useful in studying the PNS of human subjects was first suggested by the earliest papers on the response properties of single afferents (Vallbo and Hagbarth, 1968; Hagbarth ct al., 1970). Spccifically, movement across the receptive field was shown most effective in evoking activity in mechanoreceptors supplying the glabrous skin of the hand. Moreover, the discharge rate was shown to increase with both the velocity and abrasiveness of stimulus objects, and to vary with the direction of stimulus motion. These observations were subsequently extended to mechanoreceptors in the human radial (Järvilehto et al., 1976) and infraorbital nerves (Johansson et al., 1988a,b).

To better understand the motion-encoding properties of human cutaneous low-threshold mechanoreceptors, we conducted a quantitative study of individual afferents supplying three skin areas of particular significance for manipulative or orofacial motor behaviors: the palm and finger area innervated by the median nerve, the dorsum of the hand innervated by the radial nerve, and the perioral region innervated by the inferior alveolar nerve. Tactile stimuli were provided by a brushing stimulator used in previous psychophysical studies to characterize human capacity to discriminate velocity and direction of motion (Essick and Whitsel, 1985a,b; Essick et al., 1988, 1989, 1991, 1992). The stimulator had also been used to study the response properties of single somatosensory cortical neurons in primates (Whitsel et al., 1972; Essick and Whitsel, 1985a,b).

In this article, it is demonstrated that many mechanoreceptors, particularly the slowly adapting type II units, discharge in a remarkably consistent manner upon replication of the same brushing stimulus to the receptive field. Furthermore, the rich temporal response pattern can be related to the forces which compress, indent and stretch the skin. It is shown that highly reliable differences are evoked in the discharge by varying the velocity at which the stimulus moves across the receptive field. Moreover, different directions of movement evoke differences in the discharge that are consistently observed upon replication of the same stimuli. In the following companion paper of this series (Essick and Edin, 1994), we continue the analysis by examining the capacity of the mean firing rate of individual mechanoreceptors to encode information about the velocity of motion and the impact of changes in stimulus direction and force on this capacity. 


\section{Materials and Methods}

Subjects. Six males (ages 23-50; mean $=31.8$ ) and eight females (ages $20-28$; mean $=21.9$ ) recruited from the University of Umeă community participated in one or more microneurography experiment conducted during a 4 month period. In nine experiments, cutaneous low-threshold mechanoreceptive afferents (hereafter referred to as mechanoreceptors or units) in the right median nerve were studied; in nine experiments, in the right radial nerve; in three experiments, in the right inferior alveolar nerve; and in four experiments, in the left inferior alveolar nerve. During each 4-6 hr long recording session, the subject was comfortably seated in an adjustable dental chair. During recordings of the median and radial nerves, the right arm was supported on a custombuilt arm rest. The hand rested on a mound of clay molded to fit either the back of the hand (for median nerve recordings) or the palm (for radial nerve recordings). No other support was required to keep the hand in the desired position. During inferior alveolar nerve recordings, the jaw was stabilized in an open position by an interocclusal bite block. Only dentally trained personnel were recruited for experiments involving the face.

Prior to every session, the microneurographic recording technique and tactile stimulation protocol were fully explained to the subject and informed consent was obtained. The experiments were reviewed and approved by the local ethical committee at the University of Umea in accord with the Declaration of Helsinki. Reimbursement was provided at SEK 50/hr.

Neurophysiological recording technique. Single-unit recordings from mechanoreceptors originating in the glabrous and nonglabrous skin of the hand were obtained from a tungsten microelectrode percutaneously inserted into the median and radial nerve, respectively (Vallbo and Hagbarth, 1968; Torebjörk et al., 1987). The body of the microelectrode was $200 \mu \mathrm{m}$ in diameter, but tapered to $5-15 \mu \mathrm{m}$ at its distal tip. The tungsten was insulated with lacquer to within $10-30 \mu \mathrm{m}$ of the tip (this provided an impedance of 100-750 k , as measured in situ at $1 \mathrm{kHz}$ ). The neural signals were amplified $(10,000 \times$; bandwidth $=0.47-5 \mathrm{kHz})$ by a probe positioned near the recording site. An uninsulated reference microelectrode was first inserted $5-10 \mathrm{~cm}$ proximal to the elbow. A small electrical current was intermittently delivered while the microneurographer manipulated the microelectrode in search of the nerve. The subject signified that the nerve had been approximated in response to short-lasting and localized paresthesias within its innervation territory. A second, insulated recording microelectrode was then carefully inserted in parallel to the reference microelectrode and was gently manipulated in small steps. A second experimenter palpated the skin within the peripheral distribution of the nerve under study until the electrical activity of a single unit was identified and isolated.

Recordings from the inferior alveolar nerve were made employing a technique similar to that described above (Johansson and Olsson, 1976; Trulsson et al., 1992). Manual handling of the microelectrode was comparable to that employed in dental practice for local anesthesia of the lower teeth. As such, the microelectrodes were inserted by an intraoral approach into the mandibular nerve proximal to its entry into the mandibular foramen (located on the medial side of the ramus of the mandible).

Stable single-unit recordings typically lasted $20-30 \mathrm{~min}$, but with a few units recording conditions were stable for more than $2 \mathrm{hr}$.

Unit characterization and classification. After a unit was isolated, calibrated nylon monofilaments (delivering $0.06,0.12,0.25,0.5,1,2$, $4,8 \mathrm{mN}$ ) were employed to locate the skin position of lowest mechanical threshold and to define the threshold force. The total region of skin over which the unit was responsive to four times the threshold force was then determined and measured. This region defined the receptive field (RF). Receptor adaptation was evaluated by the sustained application for two or more seconds of a filament that delivered at least four times the threshold force (Johansson, 1978; Vallbo and Johansson, 1978). Units that exhibited sustained discharge for the duration of the stimulation were classified as slowly adapting (SA), and otherwise as fast adapting (FA). Additionally, units were classified as spontaneously active if they exhibited a sustained discharge in the absence of external stimulation.

Based on RF size, adaptation properties, presence of spontaneous activity, and regularity of evoked response, all units were classified as SAI, SAII, FAI, or FAII using standard criteria recommended by Vallbo and others (Knibestöl and Vallbo, 1970; Johansson and Vallbo, 1983; Vallbo, 1989). As such, SAI units were recognized as slowly adapting
Table 1. Number of units isolated (and quantitatively studied)

\begin{tabular}{lllll} 
Unit class & $\begin{array}{l}\text { Nerve } \\
\text { Median }\end{array}$ & Radial & Inf. alveolar & Totals \\
\hline SA I & $6(5)$ & $5(4)$ & $9(6)$ & $20(15)$ \\
SA II & $7(5)$ & $9(8)$ & $19(11)$ & $35(24)$ \\
FA I & $11(9)$ & $12(10)$ & $11(10)$ & $34(29)$ \\
FA II & $1(1)$ & $1(0)$ & $0(0)$ & $2(1)$ \\
FA-hair & $0(0)$ & $1(1)$ & $1(0)$ & $2(1)$ \\
Totals & $25(20)$ & $28(23)$ & $40(27)$ & $93(70)$
\end{tabular}

Data represent number of units isolated and classified by nerve and class. Each number in parentheses represents the actual number quantitatively studied (see text).

afferents which exhibited a high dynamic sensitivity and highly variable interspike intervals during sustained skin indentation (Iggo and Muir, 1969; Chambers et al., 1972; Edin, 1992). In contrast to SAII units, the receptive fields of SAI units may contain more than one highly sensitive spot. SAII units exhibit a more regular discharge rate, always a single highly sensitive spot and a lower dynamic sensitivity than SAI units. FAI units were recognized as fast adapting afferents which displayed discrete receptive fields with distinct borders. In contrast, FAII units (considered to correspond morphologically to Pacinian corpuscles) have large receptive fields and respond to even remotely applied light taps. FA units associated with individual hairs (FA-hair) were differentiated from FAI units by careful exploration of the receptive field; the FAhair units respond more readily to mechanical stimulation of individual hairs than to the skin surface.

Afferent sampling bias. With the tungsten microelectrode, single unit recordings are presumably most easily obtained from large diameter axons. The functional properties of cutaneous mechanoreceptors are, however, unrelated to axonal conduction velocities and hence to axon diameter (Järvilehto et al., 1981; Mackel, 1988). Thus, whereas the sample is biased towards fast-conducting low-threshold afferents (SAI, SAII, FAI, and FAII), and against mechanoreceptive C-fibers, the sampling bias should have no bearing on the reported properties of largediameter, low-threshold afferents.

A total of 93 cutaneous mechanoreceptors were isolated and classified (see Table 1). Seventy of these were quantitatively studied with moving stimuli. For the other 23 units, either the RF was not accessible by the tactile stimulator, isolation was lost prior to data collection, or the records were later determined to be multiunit.

Tactile stimulator. Moving tactile stimuli were delivered across the $\mathrm{RF}$ by a brushing stimulator used previously in psychophysical and neurophysiological studies (see Fig. $1 A$; see also Essick and Whitsel, 1985b; Essick et al., 1988, 1989). The stimulator device consisted of a servomotor and controlling electronic circuitry. The motor shaft rotated a perpendicularly positioned brass carrying tube to which the handle of a brush was connected. Brushing stimuli were delivered in both clockwise and counter-clockwise directions at velocities greater than $10 \mathrm{~cm} /$ sec with an error $<1 \%$, at velocities between 1 and $10 \mathrm{~cm} / \mathrm{sec}$ with an error $<5 \%$, and at $0.5 \mathrm{~cm} / \mathrm{sec}$ with an error $<10 \%$. The angular position of the shaft of the motor was signaled with a resolution of $\pm 0.25^{\circ}$ and was used to determine the approximate onset and completion of cach stimulus presentation.

Stimulus trials. Each stimulus trial consisted of a brush movement over a thin Teflon plate taped to the skin (see Fig. 1 B). A $1.4 \mathrm{~cm} \times 1.4$ $\mathrm{cm}$ aperture in the plate was centered over the RF of the afferent under study and defined the precise area of skin to receive the stimuli. The edges of the Teflon plate were beveled so that the bristles moved smoothly onto and off of the skin without generating noticeable mechanical transients. The bristles of the brush were trimmed so that they moved as a coherent bundle across the skin. As such, each stimulus resembled a rectangular shaped object moving at a constant velocity onto, across, and then off of the skin. The bristles did not "dance" on the skin or move in directions or at velocities contrary to that provided by the servomotor. The width of the brush always exceeded the width of the receptive field.

Force measurements and timing. To sample the time-varying forces applied by the brush to the skin, a special mechanical interface between the shaft of the motor and the brush was designed and fabricated (see Fig. $1 B$ ). Specifically, a $35 \mathrm{~mm}$ long aluminum beam was perpendicu- 

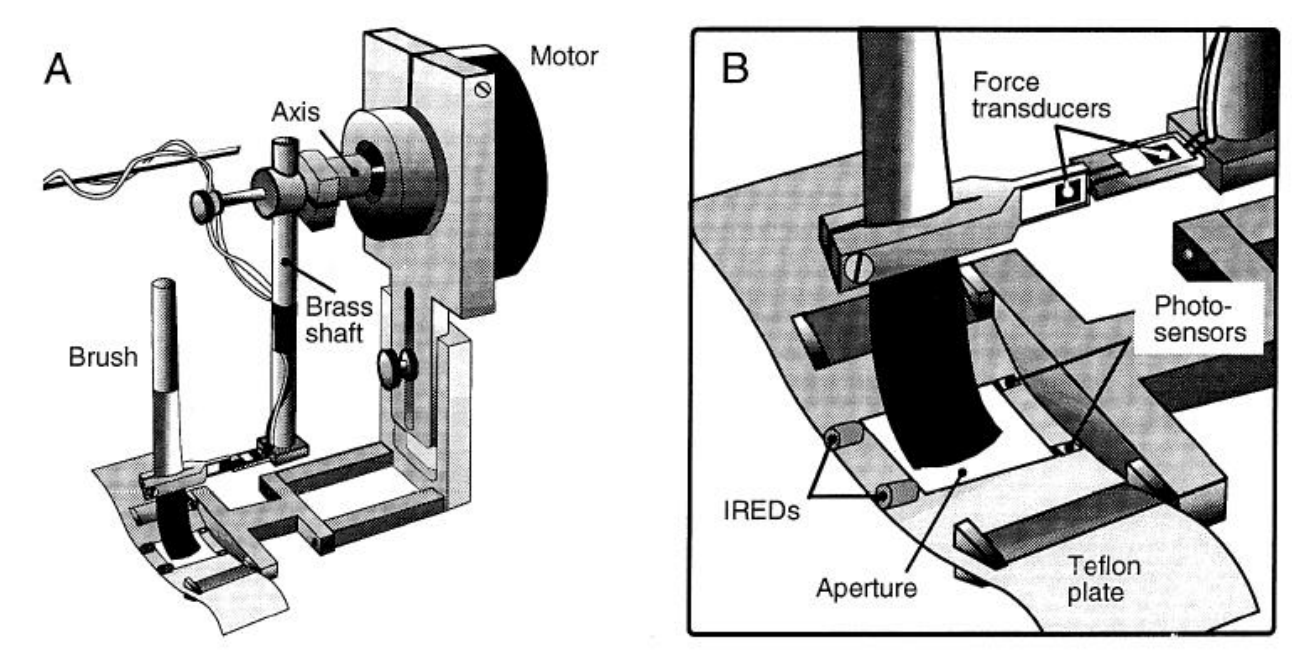

Figure 1. A, Schematic illustration of tactile stimulator. $B$, Enlarged view of the brush and force transducers in relationship to the aperture, through which the receptive field was exposed. Note the photodiodes (IREDS) and the photosensors on the edges of the aperture.

larly interposed between the brush and the carrying tube (i.e., it was parallel to both the shaft of the motor and the skin). At every instant in time, the resultant force applied by the brush to the skin could be resolved into two orthogonal components: a tangential component parallel to the surface of the skin, and a normal component perpendicular to the surface. Two pairs of strain gauges (Kyowa, KFC-2-C1-23L30, gauge factor $=2.16$ ) were mounted in quadrature on the beam to signal these two force components (bandwidth $=\mathrm{DC}-180 \mathrm{~Hz}$, peak-to-peak noise $<10 \mathrm{mN}$ ). The beam measured $5 \mathrm{~mm} \times 1.5 \mathrm{~mm}$ in the area where the strain gauges were mounted.

To allow accurate timing of the period during which the brush was in contact with the skin, infrared light-emitting diodes (IREDs) were mounted on one edge of the stimulus aperture (Fig. 1B). The light from the IREDs was received by fast responding photosensitive plates (Siemens, BP100) mounted on the opposite edge of the aperture. Thus, the light from one of the IREDs was blocked when the tip of the brush entered the aperture, and the light from the other was blocked as the brush left the aperture. As a result, the passage of the brush generated voltage depressions in the outputs of the two photosensitive plates. These depressions were separated in time as dictated by the velocity and direction of brush movement. The outputs were digitally filtered off line at $30 \mathrm{~Hz}$ and the point in time half-way between the depth of the depressions was calculated to serve as an alignment reference for all stimuli in each direction (see Fig. 6). The relation between the photosensor signals and brush contact with the skin (i.e., the times of skin onset and offset) was empirically determined and used to specify the period during which the brush bristles contacted only the skin. It was necessary to determine this period since the force records were only useful during this time interval (i.e., the bristles either were unloaded or contacted the Teflon at all other times).

Use of different brushes. Stimuli were provided by four brushes which differed in apparent stiffness and friction (see Table 2). The rationale for using more than one brush in the experiments was severalfold. First, we were particularly interested in examining the response of mecha- noreceptors distributing to the hairy skin of the hand to brushes that could be assumed to deliver relatively large tangential forces, given the recently demonstrated, exquisite sensitivity of these mechanoreceptors to lateral skin stretch (Edin and Abbs, 1991; Edin, 1992). In contrast, we were primarily interested in studying the response of mechanoreceptors distributing to the face to the soft brush used in all previous human psychophysical studies of facial sensory capacity (e.g., Essick et al., 1989, 1991). Second, it was important to determine whether gross changes in the force impacted on the peripheral encoding of stimulus velocity and direction.

Brushes of different apparent stiffness were anticipated to apply different resultant forces to the receptive field. Since the friction with the skin differed among brushes, the angle at which the resultant force was delivered differed. Thus, the relative magnitudes of the normal and tangential forces were expected to also vary among brushes.

In addition to the use of more than one brush, the magnitude of the forces delivered by the same brush was varied by changing the degree to which the bundle of bristles bent as it passed over the skin (this changed the magnitude, but not the direction of the resulting force vector). The brush extension was assessed by the distance that the brush extended through the aperture in the absence of the skin field. To categorize this measure, we referred to a minimal degree of extension (viz., $1-2 \mathrm{~mm}$ ) as a short extension; to a moderate degree of extension (viz., $2-3.5 \mathrm{~mm}$ ) as a medium extension; and greater extensions (viz., 3.5-5 $\mathrm{mm})$ as a long extension.

Figure 2 illustrates the geometric mean \pm 1 standard deviation normal force (Fig. $2 A$ ) and tangential force (Fig. $2 B$ ) delivered to the skin. Each bar represents the averaged force delivered by one of the four brushes at one extension (that is, averaged over the time interval during which the bristles contacted only the skin; cf. Fig. 6). The data from all units were pooled to generate the bar graphs. The means are convincingly rank-ordered in agreement with our a priori hypothetical ordering of the stiffness of the brushes. Similarly, the degree to which the bundle of bristles bent as it passed over the skin affected the level of applied

Table 2. Brushes used for stimulus delivery

\begin{tabular}{|c|c|c|c|c|}
\hline Description & $\begin{array}{l}\text { Bristle } \\
\text { length }\end{array}$ & $\begin{array}{l}\text { Bundle } \\
\text { width }\end{array}$ & $\begin{array}{l}\text { Percentage } \\
\text { data }\end{array}$ & $\begin{array}{l}\text { Coined name } \\
\text { for brush }\end{array}$ \\
\hline Camel's hair & $2.75 \mathrm{~cm}$ & $1.2 \mathrm{~cm}$ & 51 & Brown \\
\hline $\begin{array}{l}\text { Richeson \#9100, size } 1 / 2 \text {, nylon } \\
\text { with Plexiglas handle (England) }\end{array}$ & $1.2 \mathrm{~cm}$ & $1.7 \mathrm{~cm}$ & 7 & Stiff brown \\
\hline $\begin{array}{l}\text { Morilla \#250-F, size } 6 \text { white tal- } \\
\text { kon }\end{array}$ & $2.5 \mathrm{~cm}$ & $1.3 \mathrm{~cm}$ & 40 & White \\
\hline $\begin{array}{l}\text { Yasutomo } 1 / 2^{\prime \prime} \text { NB-78 Silverado } \\
\text { (Japan) }\end{array}$ & $1.7 \mathrm{~cm}$ & $1.3 \mathrm{~cm}$ & 2 & Stiff white \\
\hline
\end{tabular}

Data describe brushes used to deliver moving tactile stimuli across the receptive fields of the mechanoreceptors studied. As indicated by the fourth column, most of the data were collected with two brushes, a "brown brush" and a "white brush." The brushes are listed in their order of "stiffness," as subjectively determined by palpation. 

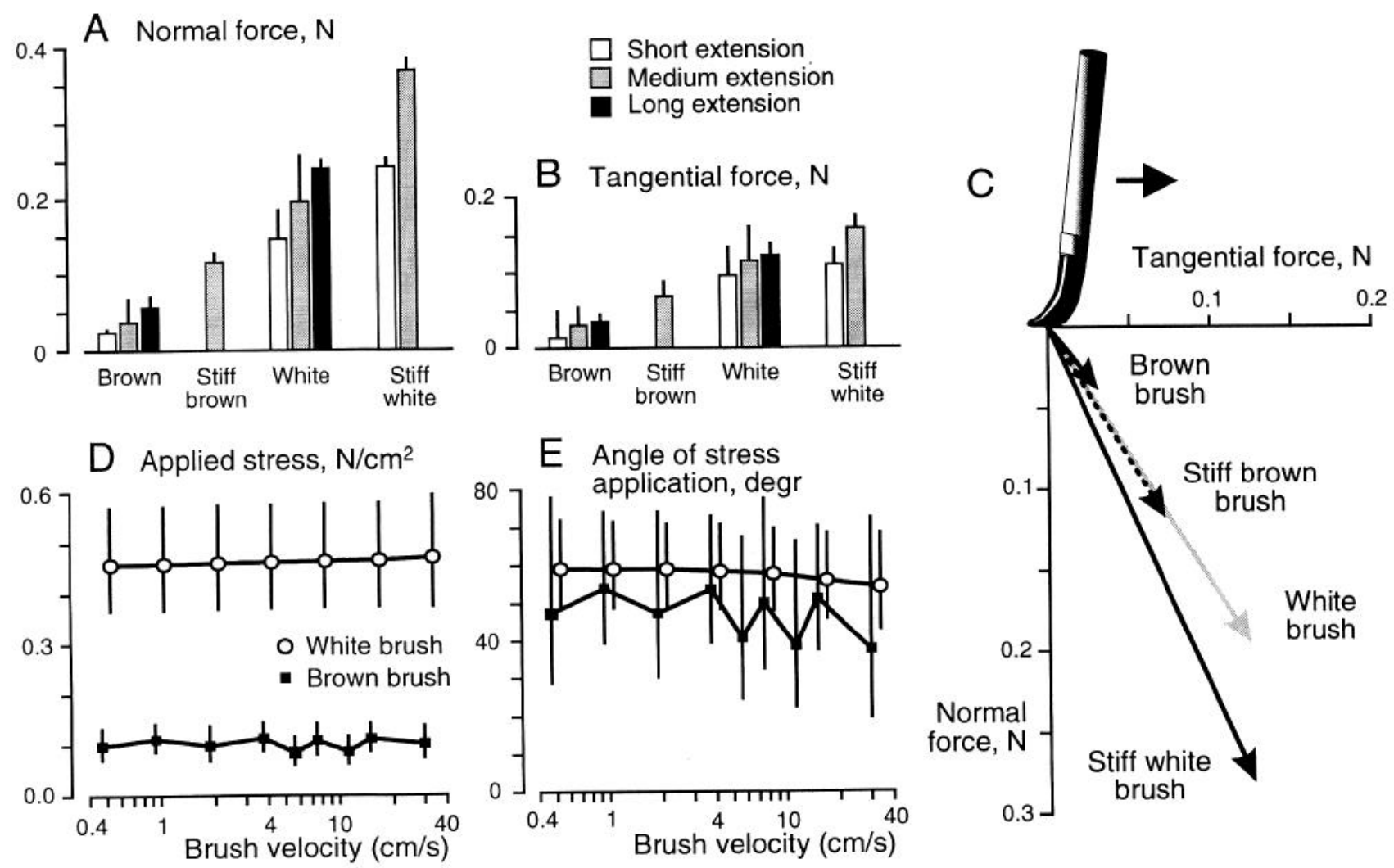

Figure 2. Geometric mean averaged normal force $(A)$ and tangential force $(B)$ applied by the four brushes across the skin. Data are shown for each extension to which the bristle were bent against the skin. Vertical bars indicate $+1 \mathrm{SD}$. $C$, The direction and magnitude of the averaged resultant force for the four brushes. Data pooled over extensions. The friction was highest with the brown brush and lowest for the stiff white brush. $D$ and $E$, The applied stress and angle of stress application, respectively, with the white and brown brush at a medium extension. Vertical bars indicate \pm 1 SD.

force in accord with our hypothesis (compare the forces exerted by each brush at different extensions). Note that the normal force varied roughly 15 -fold from $24 \mathrm{mN}$ (brown brush, short extension) to $370 \mathrm{mN}$ (stiff white brush, medium extension). Moreover, the tangential forces were approximately $60-70 \%$ of the normal forces (Fig. $2 A-C$ ). The standard deviations shown in Figure 2 do not reflect the consistency at which the same force was applied upon repetition of the same stimulus, but importantly, the total extent to which variations in all experimental factors exerted an influence on the applied force (e.g., variations in RF locations, subtle variations in brush extension and the manner in which the stimulator was positioned on the skin from run to run, stimulus velocity, direction and orientation). As such, each mean \pm 1 SD should be interpreted as the range of values most commonly observed in the experiments.

Approximately $90 \%$ of the data ( 11,116 stimulus trials) were obtained with either the white or the brown brush. Moreover, $88 \%$ of these data (9753 trials) were obtained with $2-3.5 \mathrm{~mm}$ of extension of the bristles through the aperture. Given these conditions, the brown and white brush typically delivered normal forces within the ranges $21.8-67.6 \mathrm{mN}$ and $151-257.2 \mathrm{mN}$, respectively (see Fig. $2 A$ ). The values for the brown brush (camel's hair) are consistent with those previously reported in the literature by other investigators (Franzén et al., 1984). Since most of the data were collected with these two brushes, the forces delivered by them were analyzed further.

The applied stress was calculated as the vectorial sum of normal and tangential forces divided by the total area of skin contacted by the bundle of bristles. The angle of stress application was calculated from the normal and tangential force measurements using elementary trigonometry. These two variables are plottcd as a function of the velocity of stimulus motion in Figure 2, $D$ and $E$. While the stress applied by the white brush at a medium extension $\left(464 \mathrm{mN} / \mathrm{cm}^{2}\right.$, on the average) was approximately 4.5 times that applied by the brown brush $\left(103 \mathrm{mN} / \mathrm{cm}^{2}\right.$; see Fig. $2 D$ ), the angle was approximately $10^{\circ}$ less for the brown brush $\left(46.2^{\circ}\right.$, on the average) than it was for the white brush $\left(57.6^{\circ}\right.$; see Fig. $2 E$ ). Moreover, the magnitude of the applied stress appeared relatively unaffected by stimulus velocity whereas the angle of stress application decreased slightly with velocity for both brushes. The dynamic friction between the skin and the brush is by definition the ratio of the tangential force to the normal force while the brush is moving. It was on average 0.72 for the brown brush and 0.57 for the white brush, that is, a relatively larger proportion of the total applied stress was thus directed tangentially for the brown brush than for the relatively stiffer white brush (see Fig. $2 C-E)$.

In order to determine the reliability at which the same forces were attained for the same stimulus upon replication, an estimate of the coefficient of variation was calculated for the averaged normal force and the averaged tangential force for each unit. The geometric mean estimates were $4.16 \%$ and $5.04 \%$, respectively. This reflects a remarkable degree of consistency of the same stimulus to the same receptive field. It should be noted that the coefficients of variation for the white brush were actually smaller than these mean values. Specifically, from the units studied with both brushes, it was determined that the estimates for the normal and tangential forces with the white brush were only $43 \%$ and $58 \%$ of those with the brown brush, respectively. Considered together, these findings confirm that the brushing stimulator provided a quantifiable and reliable stimulus.

Stimulation protocol. A MS-DOS operated 386 stimulus delivery system was used to control stimulus sequence, velocity, and direction. Randomized blocks of moving stimuli were typically delivered at five or seven velocities (from 0.5 to $32 \mathrm{~cm} / \mathrm{sec}$ ) in each of two opposing directions (clockwise and counterclockwise). Interstimulus intervals were 4-6 sec in length. Blocks of trials were repeated 4-10 times (median = 6 times). The data from each series of blocks constituted a run. If the unitary recording lasted long enough, the servomotor was rotated $90^{\circ}$ to permit stimulation in the two orthogonal directions. Alternatively, the unit was studied with a change in the brush (so as to increase or 

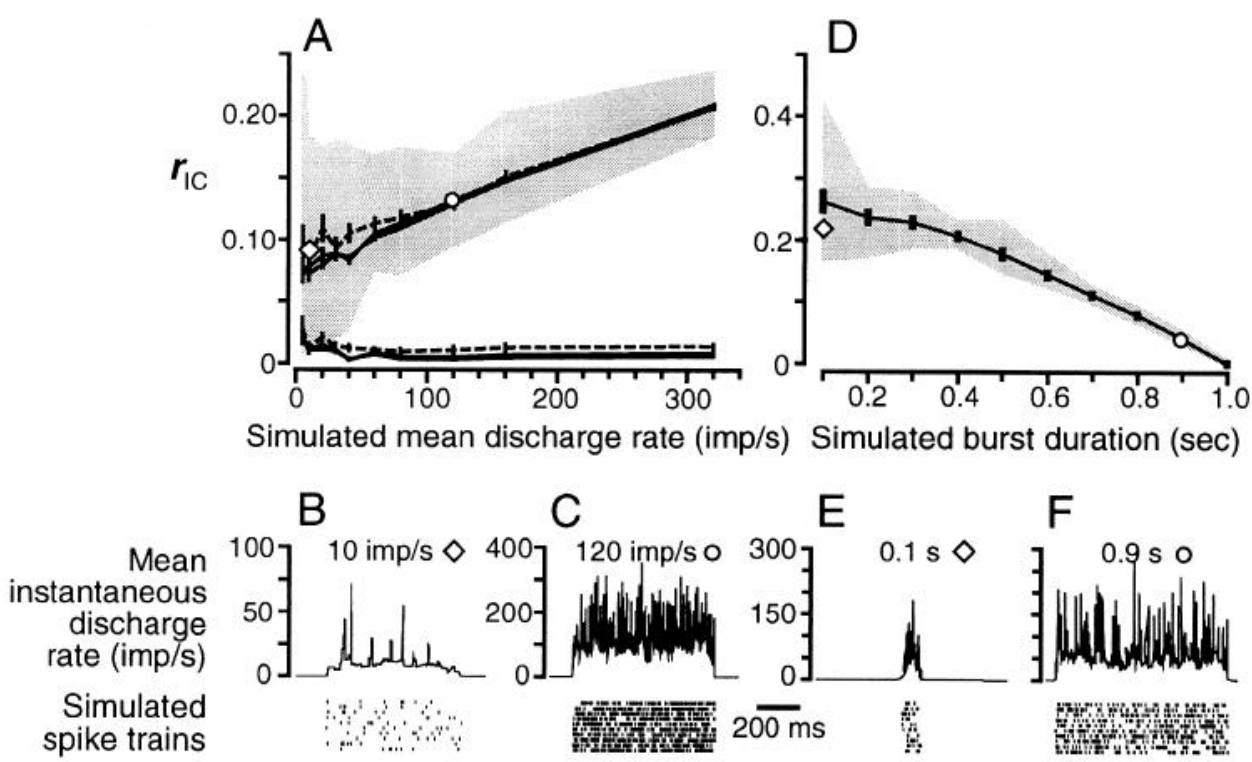

Figure 3. Twenty sets of 10 uncorrelated random spike trains were generated in a $1 \mathrm{sec}$ time window with a fixed response duration of 750 msec $(A-C)$ or from $0.1-1.0 \mathrm{sec}(D-F)$. Each vertical bar indicates the SEM, and each grayed zone, the total range of values for $r_{\text {IC }}(A, D)$. $A-C$, For mean discharge rates ranging from $5-320 \mathrm{~Hz}, r_{\mathrm{IC}}$ was calculated when the discharge rate had been sampled at 100 (dashed line), 400 , or $1600 \mathrm{~Hz}$ (solid lines). When $r_{\mathrm{IC}}$ was calculated for the $750 \mathrm{msec}$ duration of the response only (lower curves in $A$ ), $r_{\mathrm{IC}}$ was virtually zero for all mean discharge rates. If the whole $1 \mathrm{sec}$ window of sampled values was included in the calculation, $r_{\text {IC }}$ increased with the mean discharge rate. The open diamond and circle in $A$ correspond to the sample sets in $B$ and $C$ (diamond, $10 \mathrm{imp} / \mathrm{sec}$; circle, $120 \mathrm{imp} / \mathrm{sec}$ ). Subjectively in agreement, the spike trains in $C$ do appear more "similar" to each other than the trains in $B . D-F$, Spikes trains were constructed simulating response durations ranging from $0.1-1.0 \mathrm{sec}$. The discharge rates were sampled at $400 \mathrm{~Hz}$ and the mean $r_{\mathrm{IC}}$ plotted against response duration $(D)$. For uncorrelated spike trains of short duration viewed in an extended time window, $r_{\mathrm{IC}}$ approached 0.3 but was below 0.2 for response durations $>60 \%$ of the time window. Indeed, when the whole time window is considered, the spike trains in $E$ do appear more "similar" to each other than the trains in $F$. The open diamond and circle in $D$ correspond to the sample set in $E$ and $F$ (diamond, 0.1 sec response duration; circle, 0.9 sec response duration).

decrease the forces applied to the receptive field) or the dimensions of the aperture were reduced to expose less skin surrounding the RF to the stimuli.

Signal and data processing. All analog signals were sampled with 12 bits of resolution, force signals were sampled at $400 \mathrm{~Hz}$, the signals from the photosensors at $800 \mathrm{~Hz}$, and the nerve signal at $12,800 \mathrm{~Hz}$. These were accomplished by a second 386 computer which ran a custom-designed data acquisition and analysis program (sc/zoom, Department of Physiology, University of Umeá, Sweden). All signals were stored on an optical disk for off-line processing.

After each experiment, the individual nerve spikes were first retrieved from the sampled records and inspected using previously described algorithms (Edin et al., 1988). The records were then sorted by stimulus velocity and direction on a run-by-run basis.

The time of occurrence of each retrieved spike was stored and used to calculate the instantaneous discharge rate. The discharge rate at any moment was defined as the inverse of the interspike interval throughout the duration of the interspike interval. This calculated rate was then "sampled" at $800 \mathrm{~Hz}$ so that it could be treated quantitatively as other sampled signals. Importantly, the discharge rate calculated in this manner did not represent any data reduction. Since the "sampling bin" was shorter than the shortest interspike interval in the record, it was possible to reconstruct the time of occurrence of each spike (no unit ever showed instantaneous discharge rates greater than $500 \mathrm{~Hz}$ ).

Analyzing spike train similarity. From visual inspection of the recorded spike trains it was evident that afferents displayed marked differences in the degree to which replication of the same stimulus conditions evoked the same pattern of activity. Because we were unaware of any previously described method to assess spike train similarity, a quantitative method was developed. First, the instantaneous discharge rate was calculated for each spike train in a time window that spatially corresponded to a brush movement of $2 \mathrm{~cm}$ (e.g., $1 \mathrm{sec}$ window for a brush velocity of $2 \mathrm{~cm} / \mathrm{sec}$ ). Second, within this time window, the discharge rate was sampled into a 1600 element vector. The sampling frequency in the temporal domain was thus $400 \mathrm{~Hz}$ for a brush velocity of $0.5 \mathrm{~cm} / \mathrm{sec}$ and $25,600 \mathrm{~Hz}$ for $32 \mathrm{~cm} / \mathrm{sec}$. The analysis of similarity of a particular set of $n$ spike trains proceeded by creating a $1600 \times n$ matrix. Third, the intraclass correlation coefficient, $r_{\mathrm{IC}}$ (Koch, 1985), was calculated with respect to the $n$ aligned spike trains by feeding estimates from a one-factor analysis of variance (the one factor represented by the 1600 rows of the $1600 \times n$ matrix) into the equation

$$
r_{\mathrm{IC}} \cong 1-\frac{\mathrm{SS}_{\text {error }} \cdot n}{\left(\mathrm{SS}_{\text {error }}+\mathrm{SS}_{\text {row }}\right) \cdot(n-1)},
$$

in which $n$ is the number of replications, $\mathrm{SS}_{\text {row }}$ is the sum of squares over the 1600 rows, and $\mathrm{SS}_{\text {crror }}$ is the error sum of squares. Evidently $r_{\mathrm{IC}}$ approaches unity when $\mathrm{SS}_{\text {row }} \gg \mathrm{SS}_{\text {error }}$, that is, when most of the variance is explained by the rows rather than by differences among the $n$ columns (replications). In contrast, when $\mathrm{SS}_{\text {error }} \gg \mathrm{SS}_{\text {row }}$ most of the variance is attributed to differences among the replications and $r_{\mathrm{IC}}$ approaches zero. Thus, $r_{\mathrm{IC}}$ should have a value close to zero for uncorrelated spike trains and close to unity for similar ones.

The following three factors were a priori considered to potentially influence the lowest attainable level of $r_{\mathrm{IC}}$ : the ratio between the duration of the time window used for the calculations and the duration of stimulus evoked activity, the mean discharge rate during the response period, and the frequency at which the instantaneous discharge rate was sampled. Extensive simulations with generated uncorrelated spike trains were carried out to determine the sensitivity of $r_{\mathrm{IC}}$ to these factors as well as to provide reference values for interpretation of the analyses of the recorded spike trains (Fig. 3).

In a first approach, a unit was simulated to respond during a 750 msec period of a $1 \mathrm{sec}$ window (Fig. $3 B, C$ ). The simulated mean discharge rate during the response period ranged from 5 to 320 impulses per second (imp/sec). For each mean discharge rate, 20 sets of 10 spike trains were generated. For each such set, the instantaneous discharge rate was calculated and sampled at 100,400 , and $1600 \mathrm{~Hz}$. The $r_{\mathrm{IC}}$ for each set was calculated for either the entire $1 \mathrm{sec}$ time window or just the $750 \mathrm{msec}$ response period. The overall and standard error of the mean $r_{\mathrm{IC}}$ for each mean discharge rate and sampling frequency was calculated and plotted as shown in Figure $3 A$.

Several important observations were made. First, $r_{\mathrm{IC}}$ approximated zero for uncorrelated spike trains irrespective of the mean discharge 


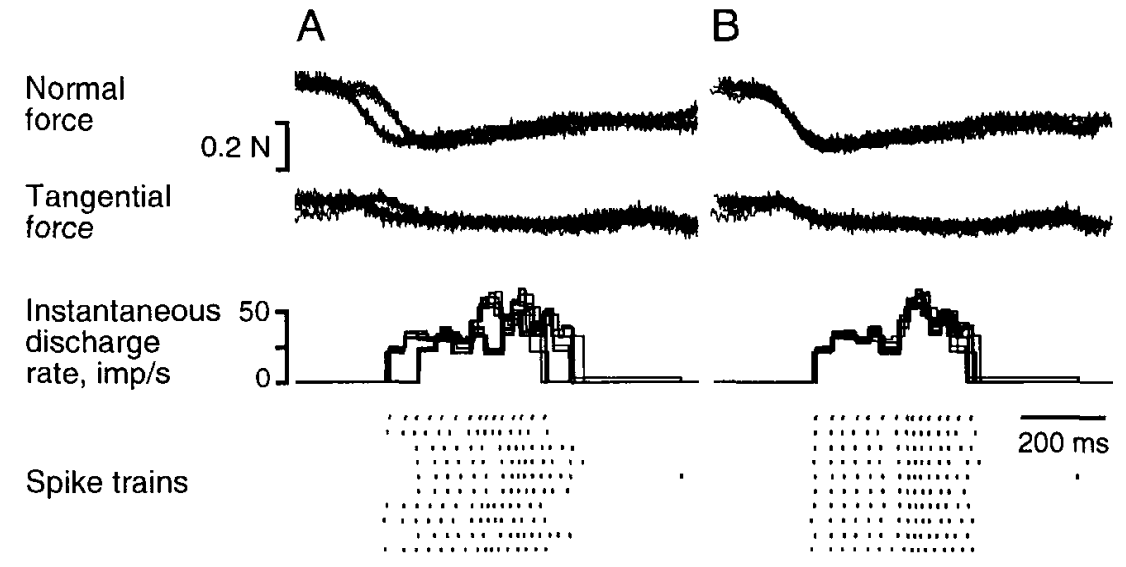

Figure 4. Spike trains evoked by 10 replications of brush stimuli moving in the proximal $\rightarrow$ distal direction at a velocity of $2 \mathrm{~cm} / \mathrm{sec}$ across the receptive field of a SAI unit on the back of the hand. The photosensor signals that were normally used for alignment were not available in this experiment (compare Fig. 6). Superimposed from top to bottom are tracings of the normal and tangential force, and the instantaneous discharge rates; spike rasters shown at the bottom. $A$, Records were initially aligned using the angular position of the stimulator shaft (accuracy $\pm 0.25^{\circ}$, not shown). $B$, Final alignment was achieved as follows. The cross-correlation between the discharge rate during each replication and the mean discharge rate of the other replications was calculated. The offset of the cross-correlation peak was successively reduced by translating each single replication. For the data shown, the maximum peak offset was $\mathbf{4 5}$ samples for the first iteration and less than one sample after the fifth and last iteration.

rate when calculated for the duration of the response only (lower set of curves, Fig. $3 A$ ). Second, $r_{1}$ increased with the mean discharge rate when calculated for the duration of the entire $1 \mathrm{sec}$ time window (upper set of curves, Fig. $3 A$ ). However, in no case did $r_{I C}$ reach values larger than 0.25 . Third, $r_{1 C}$ was not significantly influenced by the frequency at which the instantaneous discharge rate was sampled. The $r_{\text {IC }}$ calculated for the lowest sampling frequency $(100 \mathrm{~Hz}$, dashed lines, Fig. $3 A)$ while different in a statistical sense was practically identical to the means obtained with considerably higher sampling frequencies (400 and 1600 $\mathrm{Hz}$, solid lines, Fig. $3 A$ ). Moreover, as illustrated in Figurc $3 A$, the valucs for 400 and $1600 \mathrm{~Hz}$ were inseparable. Importantly, $400 \mathrm{~Hz}$ was the lowest frequency rate at which the instantaneous discharge rate was sampled in the present study.

In a second approach, the duration of the response period was varied while the simulated mean discharge rate was kept constant at $60 \mathrm{imp} /$ sec (Fig. $3 D-F$ ). For short response durations, the mean $r_{\mathrm{IC}}$ approached 0.3 but approximated 0.0 for response durations close to the length of the $1 \mathrm{sec}$ window. That the $r_{1 \mathrm{c}}$ increased when the response duration became small fits well the intended use of this index as a measurement of similarity. The spike trains in Figure $3 E$, for instance, are indeed "similar" despite that the spikes are uncorrelated for the duration of the response.

The $r_{1 C}$ thus seems to be a useful indicator of spike train similarity. It yields results that are intuitively convincing, yet it is quitc insensitive to potentially complicating factors, such as the mean discharge rate of the spike train and rate at which the discharge rate is sampled.

Handling alignment errors. All replications were not perfectly aligned in the $1600 \times n$ matrix. This was true in particular for the first eight units for which the photosensors had not yet been installed. Although the alignment errors never exceeded 100 samples (i.e., 100 rows in the matrix), they would have decreased the accuracy of any measurement of similarity. Alignment was achieved by an iterative procedure that involved calculating the cross-correlation between single replications and the mean of all replications. The rationale for this procedure was as follows. Consider a set of identical replications that contains a single malaligned replication. The peak of the cross-correlation between the malaligned replication and the mean of all replications will appear at a certain offset from zero, whereas for the rest of the replications the offset of the peak will be close to zero. If the malaligned replication is translated in a direction that reduces the offset of the cross-correlation peak, the set gradually becomes better aligned. A similar reasoning can be applied to a set in which all replications are similar but not identical. In the $1600 \times n$ matrix, each vector was translated to reduce the offset of the cross-correlation peak and the mean of the remaining vectors until no vector had to be translated more than one element. To illustrate the effect of this alignment procedure, consider the records in Figure $4 \mathrm{~A}$. These were aligned using the signal on from the angular measuring device on the stimulator (see above), but because of its limited resolution the records were incorrectly aligned. This is evident from both the superimposed records of the forces and the instantaneous discharge rates. In Figure $4 B$, the records are shown after the realignment. Note that both superimposed force and discharge rate records are now clearly well aligned.

\section{Results}

Quantitative data were obtained from 70 cutaneous, low-threshold mechanoreceptors in the human median nerve (20 units), radial nerve (23 units), and inferior alveolar nerve (27 units). The response of each mechanoreceptor to stimulus motion at multiple velocities (median $=7$ ) in each of two opposing directions was obtained. Ten units were, in addition, studied with opposing directions delivered along a second orientation across the receptive field, orthogonal to the first. Moreover, 16 units were studied with more than one brush or bristle extension against the skin. Finally, 15 units were stimulated through more than one aperture so as to vary the total area of skin exposed to the moving brush. All together, a total of 156 runs of singleunit data were collected constituting the neural response to 12,323 stimulus trials. Force data were available for 62 of the 70 quantitatively studied units. (Fabrication of the force transducing circuitry was not completed until after the first eight units had been studied.) Moreover, force data obtained during runs in which the aperture was reduced in size were discarded because all bristles of the brush were not on the skin in these trials.

\section{Description of quantitatively studied units}

Roughly half of the afferents were slowly adapting (15 SAI and 24 SAII) and half were fast adapting (29 FAI, 1 FAII, and 1 FA-hair). Of the SAIIs, 13/24 were spontaneously active (geometric mean background firing rate $=5.5 \mathrm{imp} / \mathrm{sec}$ ). In addition, two SAI and two FAI units exhibited minimal background activity (geometric mean background firing rate $=2.5 \mathrm{imp} / \mathrm{sec}$ ). Approximately half of the spontaneously active units were located in each of the radial and inferior alveolar nerves (none were located in the median nerve).

The receptive fields of all 70 units are shown in Figure $5 A-$ $C$. Note that the receptive fields of the median and radial nerves were distributed over widely different locations on the skin 



Figure 5. Receptive fields of mechanoreceptors quantitatively studied with moving tactile stimuli in the median nerve $(A)$, radial nerve $(B)$, and inferior alveolar nerve $(C)$. Receptive fields of three mechanoreceptor classes (SAI, SAII, and FA) are shaded differently. Note that all FA units were classified as FAI with the two exceptions indicated. $D$, Geometric mean receptive field area (in $\mathrm{cm}^{2}$ ) for each class of mechanoreceptors in each of the three nerves. Receptive field area did not differ significantly among nerve or unit classes. $E$, Geometric mean receptive field threshold force (in $\mathrm{mN}$ ) for each class of mechanoreceptors in each of the three nerves. The thresholds for the mechanoreceptors in the median nerve were significantly greater than those in the inferior alveolar nerve. Vertical bars indicate $+1 \mathrm{SD}$.

regions innervated by these nerves. No units with receptive fields between the digits were studied because their fields could not be accessed with the stimulator. In contrast to the nerves of the hand, most all receptive fields of the inferior alveolar nerve were accessible to the brushing stimulator. The receptive fields were not uniformly distributed over the supply area of the mental nerve. Rather, they were concentrated on the vermilion and transitional epithelium with the hairy skin of the lower lip.

Consistent with other human microneurographic studies, the receptive fields were small circular and oval areas, only a few millimeters in diameter. In Figure $5 D$, receptive field area is summarized by nerve and class. The geometric mean receptive field area was $0.055 \mathrm{~cm}^{2}(\log \mathrm{SD} 0.49)$. For the sample, receptive field area did not vary among mechanoreceptors of different nerves and/or classes $(p=0.17)$. The thresholds as measured at the most sensitive spot within the RF are shown in Figure $5 E$. The ovcrall gcomctric mean thrcshold was $0.57 \mathrm{mN}$ (log SD 0.51). However, the thresholds for the mechanoreceptors in the median nerve (geometric mean $1.09 \mathrm{mN}$ ) exceeded those in the inferior alveolar nerve (geometric mean $0.36 \mathrm{mN} ; p<0.02$ ). No other statistically significant difference (at the 0.05 level) in receptive field size or threshold was observed among units of different nerves or classes. Note that in Figure 5, as elsewhere in this article, all fast adapting units are grouped into a common (fast adapting, FA) category. This is because only two fast adapt- ing units were not classified as type I (see Fig. $5 A, B$ ), and their responses to moving stimuli could not be differentiated from those of the FAIs.

\section{Consistency of neural response to replication of the same stimulus}

Figure 6 shows all records with the stiff brown brush (compare Table 2) moving at $1 \mathrm{~cm} / \mathrm{sec}$ in the radial-to-ulnar $(R \rightarrow U)$ direction over the receptive field of an SAII receptor. The records were aligned using the photosensor signals. The receptor was located in the glabrous skin at the junction at the middle and distal phalanx of the index finger. The data in this figure were extracted from a run comprising 280 trials with stimuli of different velocities $(0.5-32 \mathrm{~cm} / \mathrm{sec})$ delivered in both the $\mathrm{R} \rightarrow$ $\mathrm{U}$ and the ulnar-to-radial $(\mathrm{U} \rightarrow \mathrm{R})$ directions. Figure 6 serves to illustrate several general statements related to both the employed stimulation method and the response of single afferents.

First, stimuli were delivered with little variability in both velocity and applied forces: to illustrate, the force and photosensor records each represent 10 superimposed single trials. Second, the response onset of most afferents showed very little variability. The particular afferent in Figure 6 responded within a time window $<11 \mathrm{msec}$ long, a period during which the brush at most moved $110 \mu \mathrm{m}$ across the skin. (The ability of individual afferents to accurately signal the stimulus onset will be dealt with in more detail in a subsequent paper devoted to the analysis 




Figure 6. The response of an SAII mechanoreceptor was recorded to 10 replications of the same stimulus moving across the RF, located above the distal interphalangeal joint of the index finger. Velocity of motion was $1.0 \mathrm{~cm} /$ $\mathrm{sec}$ in the radial $\rightarrow$ ulnar direction. From top to bottom, sampled records of superimposed tracings of the normal $(a)$ and tangential $(b)$ forces, superimposed photodiodes outputs $(c)$, nerve signals for the first, sccond, and tenth replication $(d)$, individual spike trains $(e)$, and averaged instantaneous firing rates $( \pm \mathrm{SD}, f)$. The dotted, centrally located, vertical line $(g)$ indicates the common reference or alignment position for the records. The dashed lines $(h)$ demarcate the interval in time during which the brush bristles contacted only the skin. The $r_{\text {IC }}$ value for the set of spike trains was 0.783 . of population responses.) Third, the responses evoked by replication of the same stimulus were remarkably similar in general. The duration of the response and the discharge rates were similar from trial to trial for all receptor classes, and for slowly adapting receptors, the detailed patterns of the spike trains typically matched as well. For instance, between the 2 nd and 10th replication in Figure 6, about $20 \mathrm{~min}$ had elapsed and the reccptor had been subjected to more than 100 brush stimuli of different velocities and directions. The two spike trains were nevertheless virtually identical (compare trains 2 and 10, Fig. 6).

Response similarity was assessed by calculating the intraclass correlation coefficient, $r_{\mathrm{IC}}$, of the instantaneous discharge rate. For the spike train rasters in Figure 6 , the $r_{\text {IC }}$ value was 0.783 . As is described in the companion article of this series, both SA and FA receptors typically respond well to brush velocities at about $2 \mathrm{~cm} / \mathrm{sec}$. For this reason, responses to stimuli moving at a velocity of $2 \mathrm{~cm} / \mathrm{sec}$ were selected for a first analysis $(D \rightarrow$ $\mathrm{P}$ or $\mathrm{L} \rightarrow \mathrm{M}$ directions; $10 \mathrm{SAI}, 7 \mathrm{SAII}$, and $30 \mathrm{FA})$. For units tested with more than one brush or brush extension, a run was identified for the analysis based on the similarity of the applied forces to those of runs obtained from other units.

The two most striking findings are readily observed in Figure $7 A$, which shows the means and cumulative distributions of $r_{\mathrm{IC}}$ by nerve and unit type. In all cases the $r_{\mathrm{IC}}$ was calculated for the duration of the longest response among the replications. First, the SA units as a group displayed significantly larger $r_{\mathrm{IC}}$ than the FA units (Kolmogorov-Smirnov, $p=0.0027$ ). This difference was most pronounced for units located on the hand. The SAII units in the hand exhibited particularly high $r_{\text {IC }}$. Second, the $r_{1 \mathrm{c}}$ was generally lower and showed a larger variability among units recorded from the inferior alveolar nerve.
Figure $7 B$ illustrates the range of responses for units from the hand and the face. The units were chosen from the lower and upper range of the distribution of $r_{\mathrm{IC}}$. The $r_{\mathrm{IC}}$ values were found to correspond well to the impression obtained from inspecting the raster plots and the plots of the discharge rates: units with markedly variable responses to the replicated stimulus showed low $r_{\text {IC }}$ values, whereas the units which showed practically identical spike trains yielded $r_{\mathrm{IC}}$ values close to unity. Because not even the units with the lowest $r_{\mathrm{IC}}$ approached the range of $r_{\mathrm{IC}}$ values of simulated uncorrelated spike trains (compare Fig. $3 A$ ), it was concluded that even the units showing the highest variability (e.g., FAI unit 17-4 in Fig. $7 B$ ) displayed significant similarities from replication to replication.

The $r_{1 C}$ analysis was extended to all velocities employed. The differences in $r_{1 \mathrm{C}}$ among nerves and unit types observed at the $2 \mathrm{~cm} / \mathrm{sec}$ brush velacity were consistently observed at all brush velocities (Fig. 8). Throughout the velocity range tested, SA afferents as a group displayed a higher consistency in their responses than FA units. Furthermore, whereas the consistency in the response was similar at all velocities for the SA units, FA units showed an increased consistency in the middle range of velocities $(4-16 \mathrm{~cm} / \mathrm{sec})$. Finally, afferents recorded from hand nerves showed higher response consistencies than afferents of the same class recorded from the inferior alveolar nerve.

\section{Differential sensitivity to the phases of the complex mechanical stimulus}

Importantly noted, the response duration did not precisely coincide with the interval during which the brush was in actual contact with the receptive field (note the extent of the receptive fields as indicated in Figs. $7 B, 10-11,14)$. Indeed, some units 
A
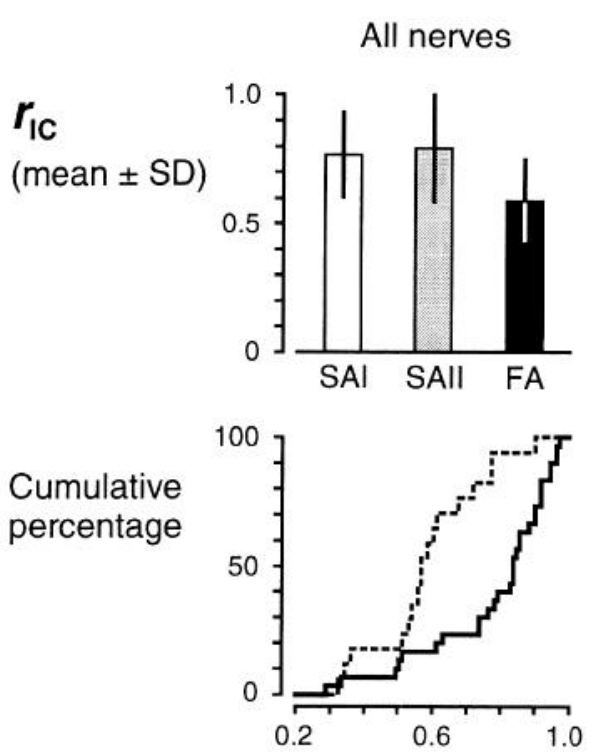

Hand nerves (radial and median $\mathrm{n}$ )
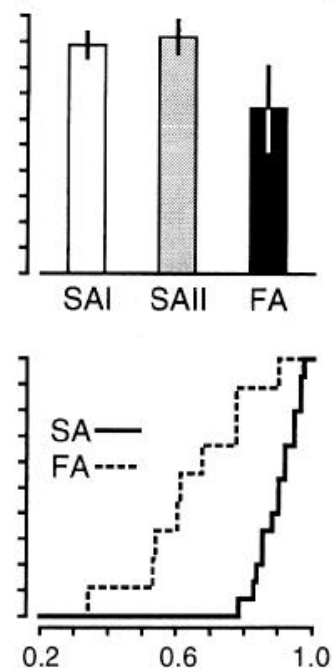

Face nerve (inf alveolar $\mathrm{n}$ )
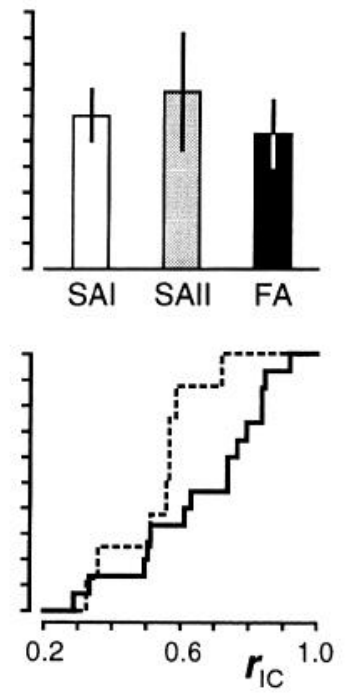

B

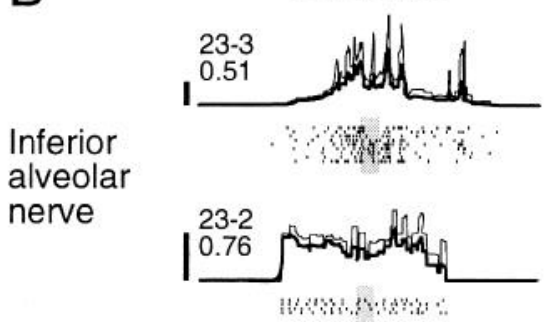

SA I units

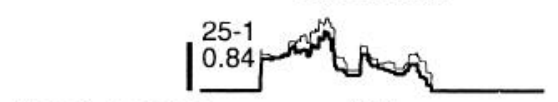

Hand nerves (radial or median $n$ )






SA II units


$(4 \mathrm{~mm})$

Figure 7. A, Mean and cumulative distribution plots of the intraclass correlation, $r_{\mathrm{ic}}$, by nerve and unit type for stimuli moving at $2 \mathrm{~cm} / \mathrm{sec}$. The brush moved in the lateral $\rightarrow$ medial direction (inferior alveolar nerve) or the distal $\rightarrow$ proximal direction (hand nerves). The $r_{1 \mathrm{C}}$ was significantly larger for SA units than for FA units from both the hand and the face. $B$, Data sets selected to illustrate the extremes of $r_{\mathrm{Ic}}$ values. For each unit, the averaged (thick line) $\pm \mathrm{SD}$ (thin lines) instantaneous discharge rates are displayed above raster plots of the spike trains. Vertical scale bars indicate $50 \mathrm{imp} / \mathrm{sec}$. The grayed area indicates the spatial extension of the receptive field. The $r_{\mathrm{IC}}$ and unit number are indicated at the top left corner of each discharge rate plot.

began to discharge before the brush had reached their receptive fields, that is, when the skin in front of the moving brush was compressed (e.g., SAI unit 25-1, Fig. 7B). Similarly, many units responded to the stretch imposed on the skin when the brush had moved beyond the receptive field (e.g., SAII unit 23-1, Fig. $7 B$ ). As a consequence, multiple peaks and troughs in the discharge rate were common. These peaks seemed to correspond to the sequential, partially overlapping phases of the mechanical stimulation: compression, indentation and stretch of the skin. With a minority of the units (e.g., SAII unit 5-1, FAI unit 204 , Fig. $7 B$ ), the response seemed to be mainly invoked by skin indentation. Other units showed brisk responses to skin compression (e.g., SAI unit 25-1, Fig. 7B); whereas still others showed evidence of responding to all three phases of the brush stimulus (e.g., SAI unit 23-3, FAI unit 19-1, Fig. 7B). The unique responses of individual mechanoreceptors thus seemed to reflect a differential sensitivity to the various components of the mechanical deformation produced by the moving brush stimulus.

\section{Effect of previous stimulus direction on response patterns}

The data for the analyses shown in Figure 7 were selected without respect to the prehistory of the stimulation, that is, about 

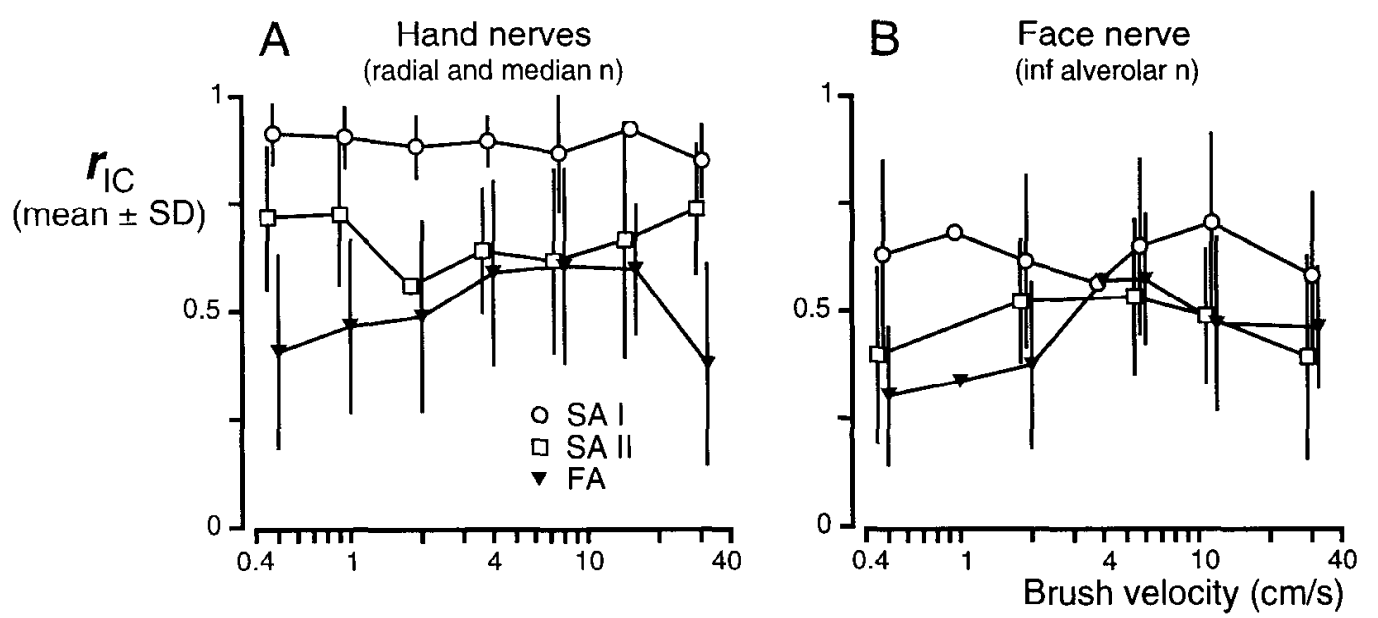

Figure 8. The mean of the intraclass correlation coefficient, $r_{\mathrm{I}}$, as a function of brush velocity for units recorded from the hand $(A)$ and the face $(B)$. Vertical bars indicate $\pm 1 \mathrm{SD}$.

one-half of the spike trains were preceded by trials with brush movements in the same direction $(\mathrm{L} \rightarrow \mathrm{M}$ or $\mathrm{D} \rightarrow \mathrm{P})$ and the other half by brush movements in the opposite direction.

It is well known that the response of cutaneous mechanoreceptors may be influenced several minutes after the cessation of a mechanical stimulus (Pubols, 1982a,b). No evidence was found in the present study, however, to indicate that the movement direction in the previous trial caused short- or long-term changes in unit responsivity. The effect of the trial immediately preceding each of those used for the $r_{\mathrm{IC}}$ analysis shown in Figure $7 B$ was assessed by comparing two subsets of data: one consisting of trials preceded by trials of the same stimulus direction and the other preceded by the opposite stimulus direction. The results of this analysis are exemplified by Figure 9 . The mean instantaneous discharge rates and the spike trains for the SAI unit 22-4 unit were virtually identical for the two groups of trials with different prehistories (see Fig. 9A-C). Thus, movement direction in previous trials exerted a negligible effect on SA units. Moreover, previous movement direction did not appear to greatly contribute to the increased variability of the FA units (see Fig. $9 D-F$; FAI unit 17-4 also shown in Fig. $7 B$ ).

\section{Response patterns for different velocities and movement directions}

The records in Figure 10 illustrate a striking observation common to almost all SA units and to many FA units. Although the dischargc rates increased dramatically with increasing brush velocities (note the vertical scale bar in Fig. 10A,D), the peaks and troughs of the rates showed a similar pattern of modulation relative to the skin surface irrespective of the stimulus velocity. The similarity was most striking at nearby velocities but with some units the spatial response profiles showed a definite resemblance even at velocities differing eightfold in magnitude (Fig. 10B). The spatial response pattern for most skin mechanoreceptors was thus preserved for stimuli moving in the same direction but at different velocities.

In contrast, the spatial responses patterns were markedly different for stimuli moving in opposite directions even though the discharge rates were often similar (though seldom identical; Fig. $10 B, E$ ). Moreover, the responses evoked by the brush at any particular spatial position on the skin did not match for opposing directions, that is, the response profile in one stimulus direction was not simply a mirror image of the response profile in the opposite stimulus direction (Fig. 10B). These observations thus extend and give further support to the conclusion presented above: The units exhibited a differential sensitivity to the components of the complex mechanical stimulus, that is, skin compression, indentation and stretch produced by the moving brush. Moreover, the discharge evoked from any given unit depends on the direction of motion. The SAII unit in Figure $10 D-F$, for example, responded briskly to the skin compression evoked by the brush when it moved in the $\mathrm{D} \rightarrow \mathrm{P}$ direction but not when the brush moved in $\mathrm{P} \rightarrow \mathrm{D}$ direction (Fig. 10E). In contrast, the SAI unit in Figure 10 responded more briskly to skin indentation and less to skin stretch when the brush moved in the $\mathrm{D} \rightarrow \mathrm{P}$ direction than when the brush moved in the $\mathrm{P} \rightarrow \mathrm{D}$ direction (Fig. 10A,B).

For 10 units, responses were recorded to brush stimuli moving in two orthogonal orientations. The responses of two of these units are shown in Figure 11. The white brush was moved over the receptive field of the FAI unit in the radial-ulnar and proximal-distal orientations (Fig. $11 A, B$ ) and the brown brush over the receptive field of an SAII unit in the medial-lateral and superior-inferior orientations (Fig. $11 C, D$ ). The responses were not only markedly different for stimuli moving in opposite directions, but also for stimuli moving in different orientations. Again, the magnitude of the responses was relatively similar in opposing directions, but the patterns were different. Moreover, there was little resemblance of response patterns to stimuli of different orientations.

\section{Response patterns for different force magnitudes}

The effect of changing the brush extension and thereby changing the total force applied by the brush to the skin was studied with 16 units. In Figure 12, the responses of two units to the brown brush are compared with those evoked by the white brush (compare Table 2). With these two brushes, markedly different magnitudes of forces were delivered to the skin as evident from the superimposed force tracings. The typical and most striking effect of increasing the force was an increase in the number of evoked action potentials (e.g., compare Fig. $12 A, C$ ). As a consequence, higher discharge rates were commonly observed with greater forces. For some units increasing the applied force also revealed previously inconspicuous or nonobserved elements in the re- 
Figure 9. The response of a SAI (A$C)$ and FAI $(D-F)$ unit to brush movements in the distal $\rightarrow$ proximal direction at $2 \mathrm{~cm} / \mathrm{sec}$ immediately preceded by stimuli in the same $(A, D)$ or in the opposite direction $(B, E)$. The mean instantaneous discharge rates were calculated for each of the two sets of spike trains and superimposed in $C$ and $F$. The vertical scale bars indicate $50 \mathrm{imp} /$ sec.
A SA I unit 22-4

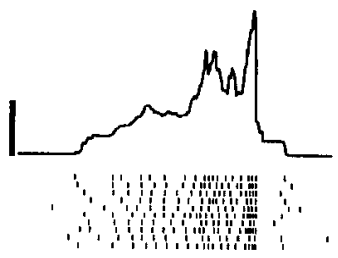

B
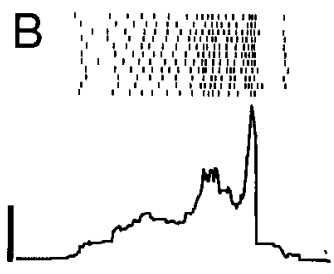

Previous stimulation in opposite direction

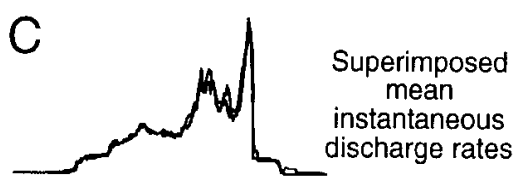

FA | unit 17-4
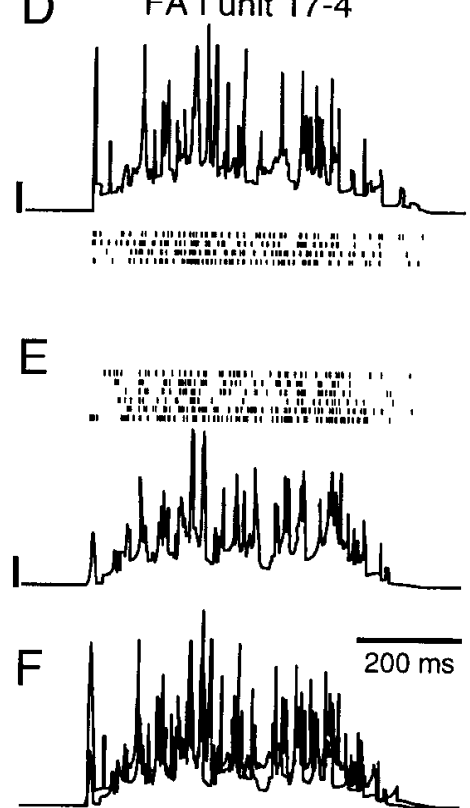

sponse profiles (Fig. 12A,C), whereas for other units the effect was primarily an accentuated modulation of the response (Fig. $12 B, D)$.

\section{Response patterns for different total areas of skin stimulated}

As noted above, action potentials were often evoked both before and after the brush was in contact with the receptive field. The majority of the receptors did not start to discharge as soon as the brush touched the skin and ceased to discharge before the brush left the skin. A few receptors, however, started to respond as soon as the brush touched the skin or did not cease to discharge before the brush left the skin. Since the receptive field was centered in the aperture and always was smaller in sizc, it seemed unlikely that slight reductions of the aperture size should significantly influence the receptors' responses to the brush stimuli. The effect of reducing the aperture size to expose less skin surrounding the RF was studied in 15 units. In these experiments, the dimensions of the reduced aperture always equaled or exceeded those of the RF.

The effect of reducing the aperture dimensions is exemplified by the data shown in Figure 13. Stimuli were delivered in the medial-lateral orientation across the face over a total of 1.96 and $0.25 \mathrm{~cm}^{2}$ of skin (i.e., using the $1.4 \mathrm{~cm} \times 1.4 \mathrm{~cm}$ and 0.5 $\mathrm{cm} \times 0.5 \mathrm{~cm}$ aperture, respectively). For FAI unit 19-1 (Fig. $13 \mathrm{~A}$ ) the reduction in aperture size resulted in loss of a stretchrelated response component for stimuli moving in the $\mathrm{L} \rightarrow \mathrm{M}$ direction, whereas a compression-related component was lost for stimuli moving in the opposite direction. In contrast, reducing the aperture size had little effect on the response of a second FAI unit from the same subject (Fig. 13B). For this latter unit, the notable difference with the reduced aperture was a additional burst of spikes. The burst was apparently evoked when the brush initially contacted the skin at a site in close proximity of the RF border. Thus, reducing the aperture size had either little effect or altered those components of the response evoked by the brush when in contact only with the skin surrounding the RF.

\section{Sensitivity of response patterns to unidentified stimulus attributes}

As demonstrated above, many units responded remarkably consistently to replication of well-controlled stimuli even when these trials, as in the present experiment, were mingled among hundreds of other trials of different stimulus specifications. The significance of this is not clear, but for teleological reasons it is tempting to attribute functional meaning to such consistent behavior; that is, the detailed spatiotemporal response profiles carry relevant information about the applied stimuli. For five units (one SAI, two SAII, and two FAI), the scnsitivity to stimulus attributes other than those purposely controlled was examined in the following manner. The position of the aperture was carefully noted (marked on the skin) as well as the position of the brushing stimulator in relation to the RF. After one or several runs for which either the aperture plate, the position of the aperture plate, the brush or its extension had been altered, the original experimental conditions ("set-up") were reproduced as closely as possible. An example of data obtained is provided in Figure 14. Shown are the responses of one SAII unit in the glabrous skin to the same stimuli delivered in two runs separated by about an hour in time. It is clear from Figure $14, A$ and $B$, that details of the response profiles were markedly different for the two runs. Force tracings and mean instantaneous discharge rates for the two runs have been superimposed for comparison in Figure 14, $C$ and $D$.

Such drastic differences in the details of the response pattern were observed with all five units despite the fact that the aperture plate was in the same position, that the stimulator was oriented the same way within a few degrees, that the brush delivered comparable forces, and that the brush moved with the same velocity. Furthermore, these differences were observed despite the fact that all five units showed a high degree of response consistency to the same stimulus within each run (for all units $r_{\text {IC }}>0.67$; compare Fig. 7). 
A

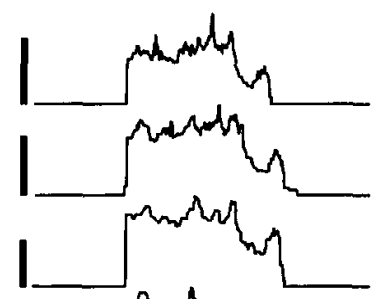

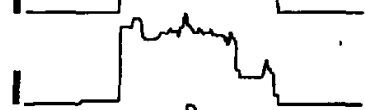

Largest RF $\varnothing$

$B$



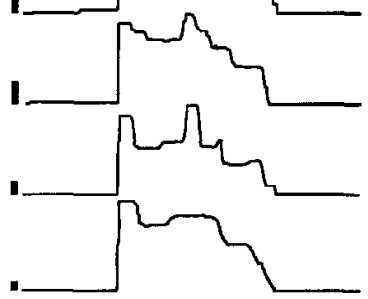

Proximal $\rightarrow$ Distal



$1 \mathrm{~cm} / \mathrm{s}$

$2 \mathrm{~cm} / \mathrm{s}$

$4 \mathrm{~cm} / \mathrm{s}$

$8 \mathrm{~cm} / \mathrm{s}$

$16 \mathrm{~cm} / \mathrm{s}$

$32 \mathrm{~cm} / \mathrm{s}$

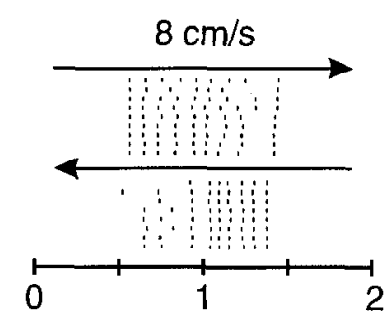

D Proximal $\rightarrow$ Distal

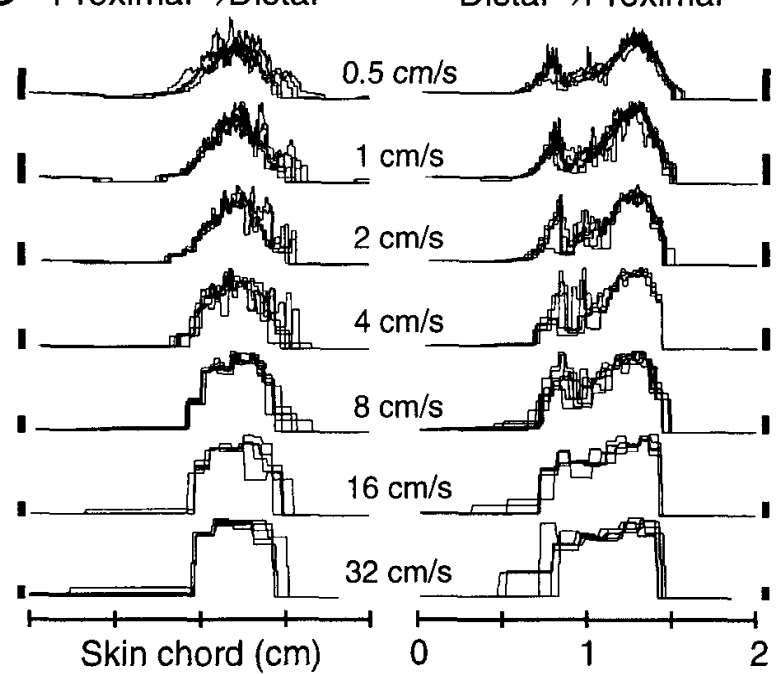

Distal $\rightarrow$ Proximal
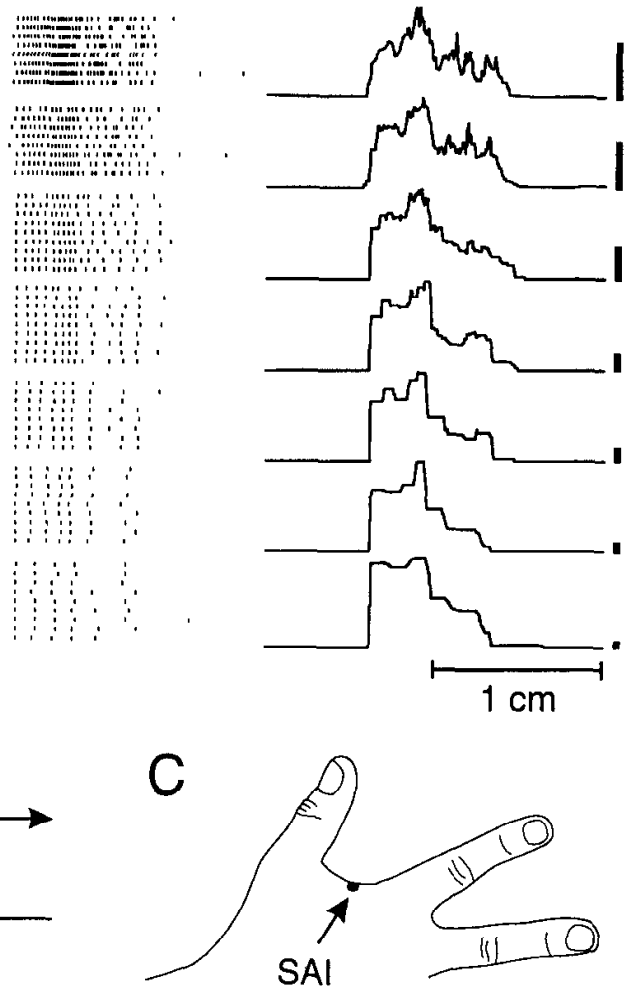

SAI

$E$

E $\quad 1 \mathrm{~cm} / \mathrm{s}$

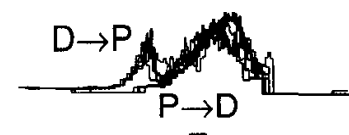

-

Largest RF $\varnothing$

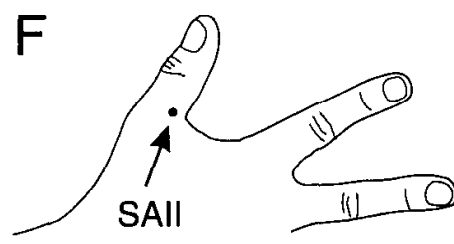

Figure 10. $A-C$, Responses and receptive field of a representative SAI unit. $A$, Mean instantaneous discharge rates and spike rasters for stimuli moving in the proximal $\rightarrow$ distal and distal $\rightarrow$ proximal directions at velocities $0.5-32 \mathrm{~cm} / \mathrm{sec}$. The vertical scale bars indicate $50 \mathrm{imp} / \mathrm{sec} . B$, Spike rasters for stimuli moving at $1 \mathrm{~cm} / \mathrm{sec}$ and $8 \mathrm{~cm} / \mathrm{sec}$ in the two opposing directions displayed in spatial coordinates so that individual spikes are aligned to the position of the brush independently of direction and velocity. $D-F$, Responses and receptive fields of a representative SAII unit. $D$, Superimposed instantaneous discharge rates with a format as in $A$. E. Superimposed instantaneous discharge rates for $1 \mathrm{~cm} / \mathrm{sec}$ plotted in time. In the distal $\rightarrow$ proximal direction there was a clear response to skin compression.

\section{Discussion}

Most previous investigators who have employed moving tactile objects have been mainly interested in the encoding of surface features rather than of the velocity and direction of the relative motion between the object and skin (Johnson and Lamb, 1981; LaMotte and Whitehouse, 1986; Goodwin and Morley, 1987a,b; LaMotte and Srinivasan, 1987a,b; Morley and Goodwin, 1987; Srinivasan and LaMotte, 1987). In their experiments, the objects were made to contact the primate fingertip in a manner so as 
Figure 11. $A$ and $B$, Responses and receptive field of a representative FAI unit. $A$, Mean instantaneous discharge rates to $2 \mathrm{~cm} / \mathrm{sec}$ brush movements in the radial $\rightarrow$ ulnar $(R \rightarrow U)$, ulnar $\rightarrow$ radial $(U \rightarrow R)$, distal $\rightarrow$ proximal $(D$ $\rightarrow P$ ), and proximal $\rightarrow$ distal $(P \rightarrow D)$ directions. $B$, Spike rasters represented in spatial coordinates: each dot represents the position of the brush on the skin when a spike was evoked. $C$ and $D$, As in $A$ and $B$ but for a SAII unit and stimuli in the medial-lateral and superior-inferior orientations.
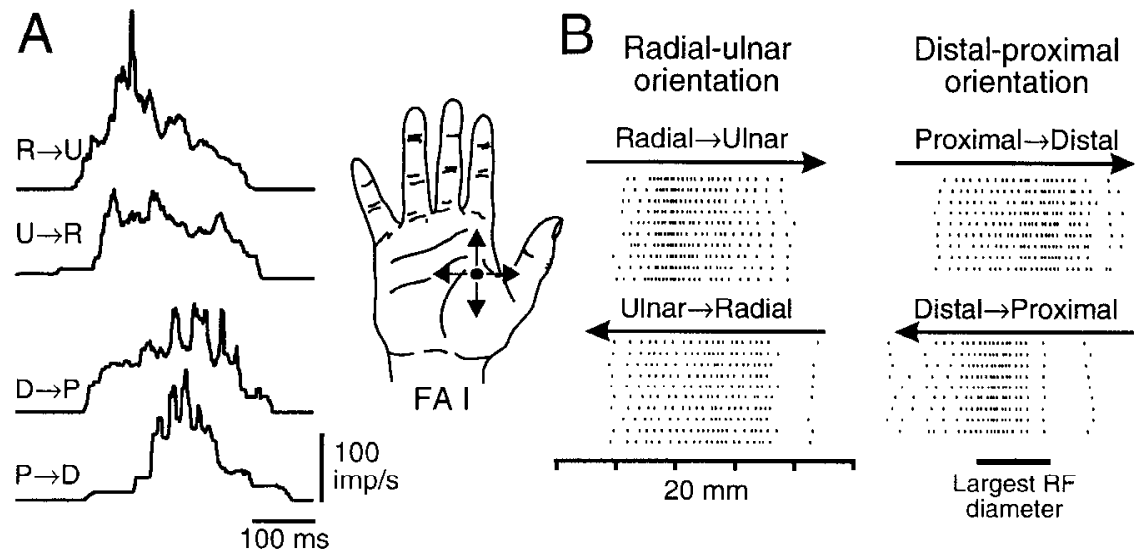
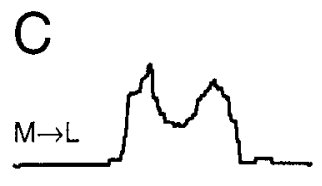

D Medial-lateral orientation

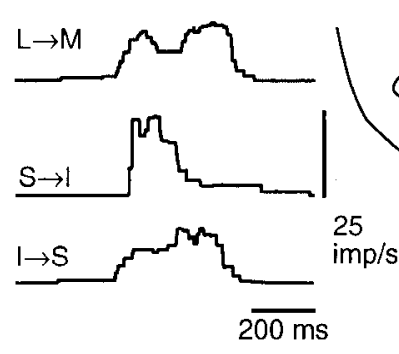

Medial $\rightarrow$ Lateral

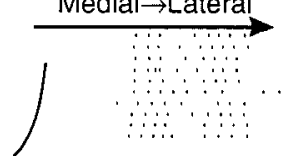

Superior-inferior orientation
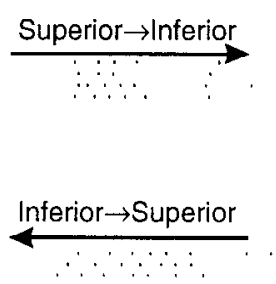
diameter to simulate the mode of stimulation that normally occurs during manual explorative and manipulative behaviors. During this mode of stimulation, whether natural or simulated, a large proportion of a relatively fixed population of receptors is engaged to encode information about adjacent areas of the surface in temporal succession (Darian-Smith and Oke, 1980; DarianSmith et al., 1980; Phillips et al., 1990, 1992). A rather different mode of stimulation is exemplified by an edge (Hyvärinen and Poranen, 1978; Costanzo and Gardner, 1980), stylus (Langford et al., 1973; Norrsell and Olausson, 1992; Olausson and Norrsell, 1993), or brush (as in the present study; Whitsel et al., 1972; Essick and Whitsel, 1985a) moving across the skin. In this case the skin area influenced by the stimulus changes with time and so must the population of receptors.

Given the different manner in which peripheral afferents are engaged by these two modes of relative movement, it is reasonable to hypothesize that there must also be differences in their central neural processing. Moreover, for each mode, movement-related information may be provided by more than one peripheral mechanism. For example, for real movement across the skin, (1) the patterns of discharge evoked in individual mechanoreceptors, (2) the intensities at which the population of excited receptors discharge, (3) the spatial distribution of receptors activated at any instant in time and changes in the distribution over time, and (4) the times at which the receptive fields along the path of motion are successively activated, all represent hypothetical encoding mechanisms. In fact, the present work was motivated in large part by the desire to obtain data bearing on these possibilities.

In this and the following companion article (Essick and Edin, 1994), we show that information about velocity and direction is available in the stimulus-evoked discharges of the population of excited receptors (viz., by a within-fiber code or one that does not depend on either the spatial locations of the receptive fields or their spatiotemporal sequence of activation). That this information is, in fact, sufficient to specify stimulus velocity and direction will be shown in a subsequent report. Moreover, the different hypothetical population-encoding schemes for moving tactile stimuli in humans will be addressed (including acrossfiber schemes that depend solely on the spatial locations of the receptive fields or their spatiotemporal sequence of activation).

Human cutaneous low-threshold mechanoreceptors respond in a highly consistent manner to moving tactile stimuli

Three main results from this study are that (1) human lowthreshold cutaneous mechanoreceptors in the glabrous and hairy skin of the hand and face respond in a highly consistent manner to replication of the same moving brush stimulus, (2) the discharge of the individual mechanoreceptor exhibits highly reliable differences to stimuli moving at different velocities, and (3) different directions of movement evoke differences in the discharge that are consistently observed upon replication of the same stimuli. With the very same stimulus employed in the present study, a similar finding was reported for the mechanoreceptors innervating the hairy skin of the monkey hindlimb (Whitsel et al., 1972). Indeed, several studies conclude that cutaneous mechanoreceptors exhibit highly consistent responses when subjected to replication of well-controlled mechanical stimuli. Hair receptors in the cat, for instance, demonstrate a high degree of consistency in their response from one replication to the next to air-jet stimuli (Goldfinger and Amassian, 1980; 

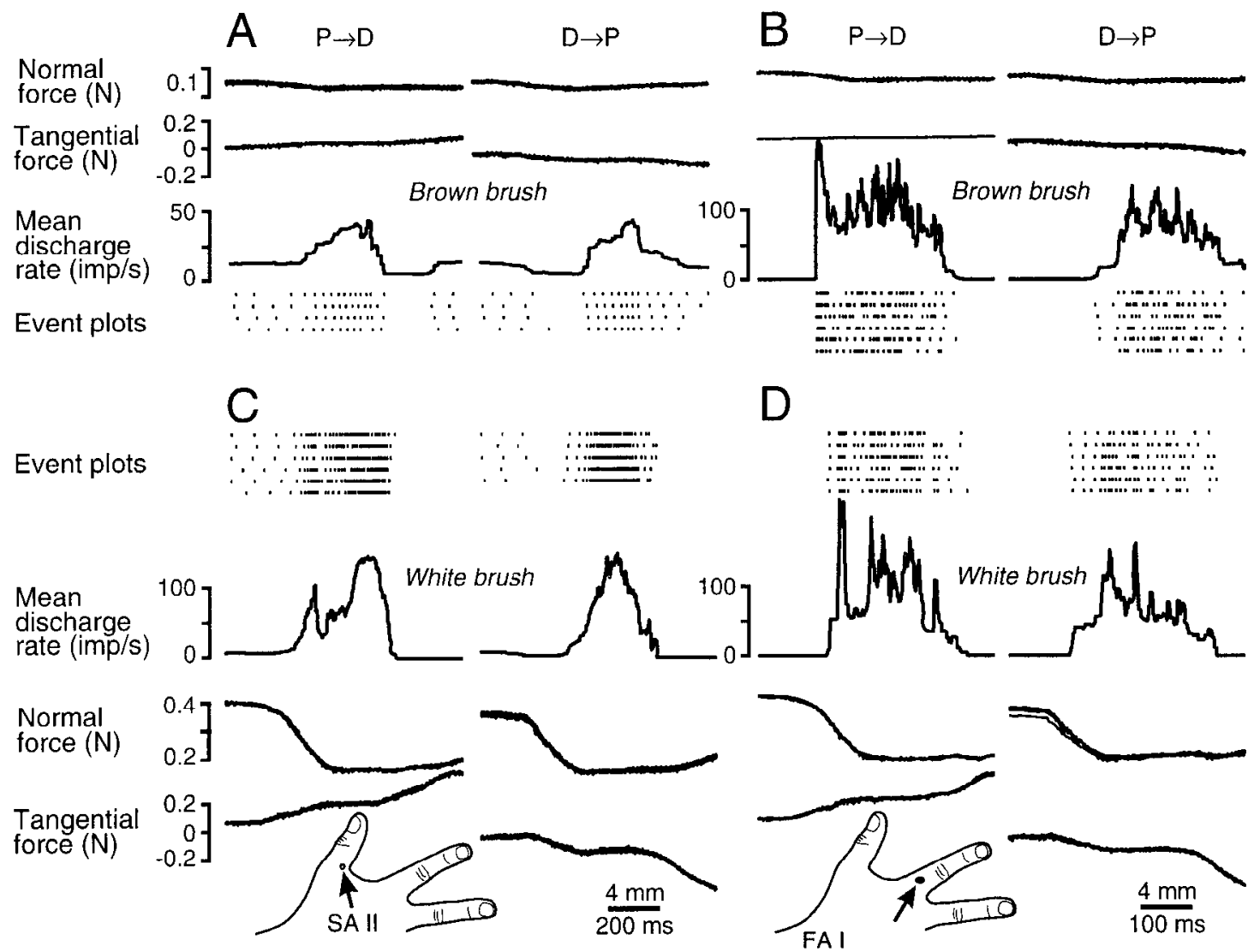

Figure 12. Effect of applied stress on the response to moving brush stimuli in the proximal $\rightarrow$ distal $(P \rightarrow D)$ and distal $\rightarrow$ proximal $(D \rightarrow P)$ directions. $A$ and $B$, Responses to the brown brush $(A, B)$ and white brush $(C, D)$ in a SAII $(A, C)$ and FAI unit $(B, D)$. The marked differences in applied forces are evident from the superimposed tracings of each of the normal and tangential forces. Increasing the applied stress caused a marked increasc in the mean discharge rates of the SAII unit $(A, C)$ but mainly influenced the magnitude of the peaks and troughs in the FAI response $(B, D)$.

Ray et al., 1985). Similarly, when embossed dot arrays are swept repetitively over the glabrous skin of the human fingertip, SAI and FAI receptors respond in such a consistent manner that the dot pattern can be reconstructed from spatial event plots of their discharge (Phillips et al., 1990, 1992). Likewise, a response profile that varies little from replication to replication is evoked in monkey slowly adapting receptors by a step stroked across the fingerpad (LaMotte and Srinivasan, 1987a). The remarkable degree of consistency in the periphery stands in marked contrast to that observed for many primate motion-sensitive, somatoscnsory cortical neurons (Hyvärinen and Poranen, 1978; Costanzo and Gardner, 1980; Essick and Whitsel, 1985b; Lee and Whitsel, 1992).

It should be noted that even those slowly adapting skin receptors that responded in a highly reproducible fashion to the brush stimuli appeared quite 'noisy' upon manual stimulation. That is, when the skin was manually poked or stroked all skin receptors responded in a seemingly irregular and inconsistent manner. Our interpretation of this apparent paradox is that these receptors encode mechanical stimuli with such high fidelity that they faithfully reflect even subtle irregularities in stimulus delivery.

Although most afferents showed consistent responses to moving tactile stimuli, there was a substantial number of units, in particular among the FA units, that were exceptional in this regard as evidenced by relatively low $r_{\mathrm{IC}}$ values (Fig. 7). This does not necessarily imply, however, that FA units are more "noisy" than the SA units. Indeed, an equally plausible interpretation is that the stimulus parameters rigorously controlled in the present experiments, that is, the brush velocity and the applied forces, did not represent the most important stimulus features for these skin mechanoreceptors. Clearly, there were perceptually important mechanical events at the brush-skin interface that were not reflected in the stimulus parameters we investigated. Texture information, for example, enabled the subjects to readily differentiate the stroke of the brush from that of a pencil. However, such information was not represented in the measured forces. It is plausible that movements of individual bristles on the skin might thus have been a significant stimulus features for FA units as well as some SAI units, whereas it likely played no or little role in the response of SAII units.

A similar reason may explain why mechanoreceptors supplying the face appeared to exhibit a lower degree of consistency than mcchanorcceptors from the hand (Fig. 7). The facial skin, particularly in areas close to the mouth, is pliable and easily deformed. It was indeed our observation that the skin was visibly deformed by the brush during many runs. It seems reasonable to assume that subtle variations in these deformations contributed to variations in the receptor response. Thus, we do not claim that facial mechanoreceptors per se are less consistent than mechanoreceptors in the glabrous or nonglabrous skin of the hand. 
Figure 13. Effect of reducing the aperture size on the responses of two FA units. $A$, Left panels show responses to stimuli in the medial $\rightarrow$ lateral $(M \rightarrow$ $L$ ) direction; the right pancls, the $L \rightarrow$ $M$ direction. The upper half of the figure illustrates the mean instantaneous discharge rate and the spike train raster plots for stimuli delivered across the 1.4 $\mathrm{cm} \times 1.4 \mathrm{~cm}$ aperture, the lower half, corresponding data for the $0.5 \mathrm{~cm} \times$ $0.5 \mathrm{~cm}$ aperture. With the smaller aperture the response components related to compression were eliminated for stimuli in the $M \rightarrow L$ direction, and to stretch for stimuli in the $L \rightarrow M$ direction. $B$, Format as in $A$, but for another FAI unit. Reducing the aperture size resulted in an initial burst of activity most prominent for stimuli in the $L \rightarrow$ $M$ direction (see asterisks). This additional response was probably related to the initial contact of the brush with the skin. The largest diameter of the RF was $4 \mathrm{~mm}$ and thus close to the border of the aperture. Vertical scale bars correspond to $50 \mathrm{imp} / \mathrm{sec}$ in $A, 100 \mathrm{imp} /$ $\sec$ in $B$.



\section{Relationship between mechanical stimulus and response patterns}

A moving tactile stimulus consists of a complex sequence of mechanical events. For example, when a brush moves across the skin, the skin in front is compressed, the subjacent skin is indented and the skin behind is stretched. As a result, a mechanoreceptor located in the movement path is subjected to partially overlapping phases dominated by compression, indentation or stretch. Indeed, most receptors we studied responded to skin stimulation even when the brush was not in direct contact with the receptive field.

These findings stand in sharp contrast with those studies in which moving tactile stimuli were only simulated. Although human studies have not been conducted to date, an air-jet stream (Goldfinger and Amassian, 1980) and Optacon device (Gardner and Palmer, 1989) have been employed to study cutaneous afferents in the cat and monkey, respectively. Both types of stimuli lack the tangential force components that are obligatory with most naturally occurring moving stimuli. As a result, application of these stimuli effectively maps out the receptive field in a manner similar to that observed from use of von Frey hairs. Peaks in the discharge correspond to particularly sensitive parts of the receptive field ("hot spots"; Goldfinger and Amassian, 1980). Receptive fields studied with the Optacon most often exhibit uniform sensitivity, with one spike typically evoked by every cycle of the vibrating probes (Gardner and Palmer, 1989). Accordingly, the spatial response profiles generated in opposing directions by these "frictionless" stimuli are remarkably similar when aligned to common spatial loci.

In contrast, with a moving brush the response to stimuli moving in opposite directions are typically quite different and cannot be made to appear the same by any simple temporal rotation or realignment. A similar finding has been reported for SA receptors in the glabrous skin of the monkey digit using smoothly graded stepped surfaces stroked across the skin (LaMotte and Srinivasan, 1987a). Furthermore, there is no simple correspondence between the discharge pattern and those features of the receptive field demonstrated by purely indenting stimuli (e.g., "hot spots," cf. LaMotte and Srinivasan, 1987a).

\section{Mechanoreceptors display differences to stimuli that move at different velocities and directions}

With increasing brush velocity, the rate of change of the stretching, compressing and indenting force components all increase. This fact paired with the pronounced dynamic sensitivity of both FA and SA units provides a simple and yet convincing explanation as to why practically all units displayed an increased discharge rate with increased brush velocity. That the underlying mechano-electrical events are similar even though they occur at different rates at different velocities, is suggested by the striking preservation of the details of the spatial discharge pattern. 




Figure 14. Between the first and eighth run, the Teflon plate had been repositioned and the brush had been changed and replaced. The experimental conditions in $A$ were replicated as closcly as possible in $B$ by adopting the same position of the Teflon plate and using the same brush and brush extension. Despite that the controlled stimulus parameters (force, brush position, velocity, and direction) were the same, the unit consistently responded differently in the two runs. Brush velocity was $2 \mathrm{~cm} / \mathrm{sec}$ and the movement directions were distal $\rightarrow$ proximal $(D \rightarrow P)$ and proximal $\rightarrow$ distal $(P \rightarrow D)$ as indicated. $C$ and $D$, For comparison, force tracings and mean instantaneous discharge rates were superimposed for each of the two movement directions.

Most low-threshold mechanoreceptors, however, exhibit distinct differences in response to brush stimuli moving in different directions even at the same stimulus velocity. These differences are most pronounced in the spatial discharge patterns. Consistent directional differences in discharge intensity (e.g., mean firing rate) are also observed, but they are often relatively subtle. Directional differences in the discharge may be explained by asymmetrical sensitivity to stretch or compression with respect to the orientation of the stimulation. Indeed, many human SAII units in the glabrous skin exhibit differential sensitivity to stretch in opposite directions (Johansson, 1978). The information provided by individual skin receptors is thus, in principle, able to differentiate not only between stimuli moving at different velocities but also between stimuli moving in different directions.

\section{Implications for the encoding of information about tactile motion}

The ability to appreciate the direction of a moving tactile stimulus is greatly enhanced when the stimulus applies tangential forces, as when, for instance, a brush or a stylus is moved across the skin (Gould et al., 1979; Bender et al., 1982; Norrsell and Olausson, 1992). Indeed, subjects readily detect the direction of skin stretch applied to discrete points on the skin (Halpern, 1949). Hence, we must conclude that the skin mechanoreceptors convey useful movement-relevant information as a result of the forces applied tangential to the skin. But why does the addition of skin stretch and compression improve psychophysical performance?

The richness and the consistency of the response patterns evoked by the moving brush stimuli invite attributing functional significance to these patterns; that is, the details of the patterns provide useful information about tactile events including the direction of motion (compare Fig. 11). For several reasons, however, we are reluctant to yield to this temptation. First, this explanation looses most of its appeal when one considers the computational demands on a putative neural system responsible for extracting information from the detailed discharge patterns of individual receptors. Such a system must be able to process the discharge pattern in the spatial rather than the temporal domain since the patterns evoked at different stimulus velocities are similar only when expressed as a function of position on the skin. This, in turn, implies a computational algorithm that is sensitive to the time of occurrence of individual spikes but insensitive to the time interval between spikes. Whereas such computations are readily carried out on a computer when the actual stimulus velocities are known (compare Fig. 11), we are unaware of any neural mechanisms that could accomplish this feat. 
The second reason we are reluctant to attribute an important functional role to the detailed temporal pattern for the encoding of brush direction is that a seemingly insignificant modification of the stimulus produces a drastic change in the response patterns (compare Fig. 14). It is difficult to imagine any neuronal processing scheme that could map distinctly different response patterns to almost identical stimulus parameters, that is, to one direction of movement.

A more attractive explanation as to why the skin stretch that normally accompanies movements improves psychophysical performance on direction discrimination tasks is that the lateral stresses and strains simply recruit additional receptors surrounding the moving stimulus (Gould et al., 1979). Moreover, the rate at which the population is recruited as well as its spatial distribution is dictated by the velocity and direction of motion, respectively. Accordingly, the accuracy with which the stimulus can be specified is predicted to be greater when a large population of afferents is activated in a particular spatiotemporal sequence than when a small population is activated directly under the stimulus. In this view it is not necessary to assign any functional role to the directional asymmetries in the discharge patterns exhibited by the individual strain-sensitive receptors. The consistency of the discharge patterns upon stimulus replication is thus considered an epiphenomenon: In order to reliably respond with a certain number of spikes or a certain mean firing rate to a particular stimulus (i.e., with a certain intensity), the receptor is endowed with certain response characteristics that reflect the sequential compression, indentation and stretch of the skin.

That skin stretch and compression are unnecessary for the percept of tactile motion, however, is well cstablishcd. An illusion of movement is readily produced by a sequence of shortlasting, discrete indentations of the skin along a cutaneous path (Kirman, 1983; Szaniszlo et al., 1992). This has been demonstrated even in experiments for which only two widely separated stimuli were sequentially delivered (Sherrick and Rogers, 1966). Although human subjects can discriminate the direction of such stimuli (Gardner and Sklar, 1986; Craig and Baihua, 1990; Evans and Craig, 1991; Essick et al., 1992), the capacity to appreciate their velocity has received little attention (Essick et al., 1991). Consistent with the existing psychophysical observations, somatosensory cortical neurons in primates have been shown to respond to discontinuously moving stimuli in the same manner as to continuously moving natural stimuli, and to differentially signal opposing directions (Gardner, 1988; Gardner et al., 1992). Thus, it is generally accepted that central neural mechanisms can infer the direction, and probably the velocity, of moving tactile stimuli solely from the times at which the spatially distributed mechanoreceptors are activated.

That detailed discharge patterns evoked in individual mechanoreceptors are not necessary for the processing of moving tactile stimuli can be tested in psychophysical experiments. This hypothesis predicts that the ability to discern between different movement directions and velocities is the same whether the skin is stimulated with exclusively indenting stimuli (e.g., with an Optacon stimulator that does not evoke complex discharge patterns in individual afferents; Gardner and Palmer, 1989) or with stimuli involving forces tangential to skin (e.g., with a moving brush) provided that the receptor population engaged is the same. In a recent preliminary study this issue was, in fact, addressed and the prediction was fulfilled (Rath and Essick, 1992). Indeed, direction discrimination was very similar for both modes of stimulation suggesting that lateral skin stretch is sufficient but not necessary for this sensory function.

\section{References}

Bender MB, Stacy C, Cohen J (1982) Agraphesthesia: a disorder of directional cutaneous kinesthesia or a disorientation in cutaneous space. J Neurol Sci 53:531-555.

Chambers MR, Andres KH, Duering M, Iggo A (1972) The structure and function of slowly adapting type II mechanoreceptors in hairy skin. Q J Exp Physiol 57:417-445.

Costamzo RM, Gardner EP (1980) A quantitative analysis of responses of direction-sensitive neurons in somatosensory cortex of awake monkeys. J Neurophysiol 43:1319-1341.

Craig JC', Baihua X (1990) Temporal order and tactile patterns. Percept Psychophys 47:22-34.

Darian-Smith I, Oke LE (1980) Peripheral neural representation of the spatial frequency of a grating moving across the monkey's finger pad. J Physiol (Lond) 309:117-133.

Darian-Smith I, Davidson I, Johnson KO (1980) Peripheral neural representation of spatial dimensions of a textured surface moving across the monkey's finger pad. J Physiol (Lond) 309:135-146.

Edin BB (1992) Quantitative analysis of static strain sensitivity in human mechanoreceptors from hairy skin. J Neurophysiol 67:1 1051113.

Edin BB, Abbs JH (1991) Finger movement responses of cutaneous mechanoreceptors in the dorsal skin of the human hand. J Neurophysiol 65:657-670.

Ẹdin BB, Bäckström PA, Bäckström LO (1988) Single unit retrieval in microneurography: a microprocessor-based device controlled by an operator. J Neurosci Methods 24:137-144.

Essick GK, Edin BB (1995) Receptor encoding of moving tactile stimuli in humans. II. The mean response of individual low-threshold mechanoreceptors to motion across the receptive field. J Neurosci 15: 848-864.

Essick GK, Whitsel BL (1985a) Factors influencing cutaneous directional sensitivity: a correlative psychophysical and neurophysiological investigation. Brain Res Rev 10:213-230.

Essick GK, Whitsel BL (1985b) Assessment of the capacity of human subjects and S-I neurons to distinguish opposing directions of stimulus motion across the skin. Brain Res Rev 10:187-212.

Essick GK, Franzén O, Whitsel BL (1988) Discrimination and scaling of velocity of stimulus motion across the skin. Somatosens Mot Res 6:21-40.

Essick GK, Whitsel BL, Dolan PJ, Kelly DG (1989) Effects of traverse length on human perioral directional sensitivity. J Neurol Sci 93:175190.

Essick GK, Franzén O, McMillian A, Whitsel B (1991) Utilization of temporal and spatial cues to judge the velocity and traverse length of a moving tactile stimulus. In: Information processing in the somatosensory system (Franzén O, Westman J, cds), pp 341-352. London: Macmillan.

Essick GK, McGuire M, Joseph A, Franzén O (1992) Characterization of the percepts evoked by discontinuous motion over the perioral skin. Somatosens Mot Res 9:175-184.

Evans PM, Craig JC (1991) Tactile attention and the perception of moving tactile stimuli. Percept Psychophys 49:355-364.

Franzén O, Thompson F, Whitsel B, Young M (1984) Peripheral coding mechanisms of touch velocity. In: Somatosensory mechanisms (von Euler C, Franzén O, Lindblom U, Ottoson D, eds), pp 213-226. New York: Plenum.

Gardner EP (1988) Somatosensory cortical mechanisms of feature detection in tactile and kinesthetic discrimination. Can J Physiol Pharmacol 66:439-454.

Gardner EP, Palmer CI (1989) Simulation of motion on the skin. I. Receptive fields and temporal frequency coding by cutaneous mechanoreceptors of OPTACON pulses delivered to the hand. J Neurophysiol 62:1410-1436.

Gardner EP, Sklar BF (1986) Factors influencing discrimination of direction of motion on the human hand. Soc Neurosci Abstr 12:798.

Gardner EP, Palmer CI, Hämäläinen HA, Warren S (1992) Simulation of motion on the skin. V. Effect of stimulus temporal frequency on the representation of moving bar patterns in primary cortex of monkeys. J Neurophysiol 67:37-63.

Goldfinger MD, Amassian VE (1980) Response of forelimb guard hair 
afferent units to air-jet stimulation of entire receptive field. $\mathrm{J}$ Neurophysiol 44:961-1001.

Goodwin AW, Morley JW (1987a) Sinusoidal movement of a grating across the monkey's fingerpad: effect of contact angle and force of the grating on afferent fiber responses. J Neurosci 7:2192-2202.

Goodwin AW, Morley JW (1987b) Sinusoidal movement of a grating across the monkey's fingerpad: representation of grating and movement features in afferent fiber responses. J Neurosci 7:2168-2180.

Gould WR, Vierck Jr CJ, Luck MM (1979) Cues supporting recognition of the orientation or direction of movement of tactile stimuli. In: Sensory function of the skin of humans. Proceedings of the Second International Symposium on the Skin Senses (Kenshalo DR, ed), pp 63-73. New York: Plenum.

Hagbarth KE, Hongell A, Hallin RG, Torebjörk HE (1970) Afferent impulses in median nerve fascicles evoked hy tactile stimuli of the human hand. Brain Res 24:423-442.

Hall GS, Donaldson HH (1885) Motor sensations on the skin. Mind 10:557 572.

Halpern L (1949) Disturbance of dermatokinesthesis in cerebral and spinal diseases. J Nerv Ment Dis 109:1-8.

Hyvärinen J, Poranen A (1978) Movement-sensitive and direction and orientation-selective cutaneous receptive fields in the hand area of the post-central gyrus in monkeys. J Physiol (Lond) 283:523-537.

Iggo A, Muir AR (1969) The structure and function of a slowly adapting touch corpuscle in hairy skin. J Physiol (Lond) 200:763-796.

Järvilehto $T$, Hämäläinen $H$, Laurinen $P$ (1976) Characteristics of single mechanoreceptive fibres innervating hairy skin of the human hand. Exp Brain Res 25:45-61.

Järvilehto $T$, Hämäläinen $H$, Soininen $K$ (1981) Peripheral neural basis of tactile sensations in man. II. Characteristics of human mechanoreceptors in the hairy skin and correlations of their activity with tactile sensations. Brain Res 219:13-27.

Johansson RS (1978) Tactile sensibility in the human hand: receptive field characteristics of mechanoreceptive units in the glabrous skin area. J Physiol (Lond) 281:101-123.

Johansson RS, Olsson KA (1976) Microelectrode recordings from human oral mechanoreceptors. Brain Res 118:307-311.

Johansson RS, Vallbo $\AA \mathrm{B}$ (1983) Tactile sensory coding in the glabrous skin of the human hand. Trends Neurosci 6:27-32.

Johansson RS, Trulsson M, Olsson KÅ, Abbs JH (1988a) Mechanoreceptive afferent activity in the infraorbital nerve in man during speech and chewing movements. Exp Brain Res 72:209-214.

Johansson RS, Trulsson M, Olsson KÄ, Westberg K-G (1988b) Mechanoreceptor activity from the human face and oral mucosa. Exp Brain Res 72:204-208.

Johnson KO, Lamb GD (1981) Neural mechanisms of spatial tactile discrimination: neural patterns evoked by braille-like dot patterns in the monkey. J Physiol (Lond) 310:117-144.

Kirman JH (1983) Tactile apparent motion: the effects of shape and type of motion. Percept Psychophys 34:96-102.

Knibestöl M, Vallbo $\AA$ B (1970) Single unit analysis of mechanoreceptive activity from the human glabrous hand. Acta Physiol Scand 80:178-195.

Koch GG (1985) Intraclass correlation coefficient. In: Encyclopedia of statistical sciences (Kotz SL, Johnson NL, eds), pp 212-217. New York: Wiley.

LaMotte RH, Srinivasan MA (1987a) Tactile discrimination of shape: responses of slowly adapting mechanoreceptive afferents to a step stroked across the monkey fingerpad. J Neurosci 7:1655-1671.

LaMotte RH, Srinivasan MA (1987b) Tactile discrimination of shape: responses of rapidly adapting mechanoreceptive afferents to a step stroked across the monkey fingerpad. J Neurosci 7:1672-1681.
LaMotte RH, Whitehouse J (1986) Tactile detection of a dot on a smooth surface: peripheral neural events. J Neurophysiol 56:11091128.

Langford N, Hall RJ, Monty RA (1973) Cutaneous perception of a track produced by a moving point across the skin. J Exp Psychol 97: $59-63$.

Lee C-J, Whitsel BL (1992) Mechanisms underlying somatosensory cortical dynamics. I. In vivo studies. Cereb Cortex 2:81-106.

Mackel R (1988) Conduction of neural impulses in human mechanoreceptive cutaneous afferents. J Physiol (Lond) 401:597-615.

Morley JW, Goodwin AW (1987) Sinusoidal movement of a grating across the monkey's fingerpad: temporal patterns of afferent fiber responses. J Neurosci 7:2181-2191.

Norrsell U, Olausson H (1992) Human, tactile, directional sensibility and its peripheral origins. Acta Physiol Scand 144:155-161.

Olausson H, Norrsell U (1993) Observations on human tactile directional sensibility. J Physiol (Lond) 464:545-559.

Phillips JR, Johansson RS, Johnson KO (1990) Representation of braille characters in human nerve fibers. Exp Brain Res 81:589-592.

Phillips JR, Johansson RS, Johnson KO (1992) Responses of human mechanoreceptive afferents to embossed dot arrays scanned across fingerpad skin. J Neurosci 12:827-839.

Pubols BH Jr (1982a) Factors affecting cutaneous mechanoreceptor response. I. Constant-force versus constant-displacement stimulation. J Neurophysiol 47:515-529.

Pubols BH Jr (1982b) Factors affecting cutaneous mechanoreceptor response. II. Changes in mechanical properties of skin with repeated stimulation. J Neurophysiol 47:530-542.

Rath EM, Essick GK (1992) Lateral skin stretch is unnecessary for normal perioral direction discrimination. J Oral Maxillofac Surg 50: 188.

Ray RH, Mallach LE, Kruger L (1985) The response of single guard and down hair mechanoreceptors to moving air-jet stimulation. Brain Res 346:333-347.

Sherrick CE, Rogers R (1966) Apparent haptic movement. Percept Psychophys 1:175-180.

Srinivasan MA, LaMotte RH (1987) Tactile discrimination of shape: responses of slowly and rapidly adapting mechanoreceptive afferents to a step indented in the monkey fingerpad. J Neurosci 7:1682-1697.

Szaniszlo RH, Essick GK, Kelly DG (1992) Spatial/temporal requirements for tactile motion perception. J Dent Res 71:197.

Torebjörk HE, Vallbo ÅB, Ochoa JL (1987) Intraneural microstimulation in man. Its relation to specificity of tactile sensations. Brain 110:1509-1529.

Trulsson M, Johansson RS, Olsson K§ (1992) Directional sensitivity of human periodontal mechanoreceptive afferents to forces applied to the teeth. J Physiol (Lond) 447:373-389.

Vallbo $\AA B$ (1989) Single fibre microneurography and sensation. In: Hierarchies in neurology. A reappraisal of a Jacksonian concept (Kennard C, Swash M, eds), pp 93-109. London: Springer.

Vallbo $\AA$ B, Hagbarth K-E (1968) Activity from skin mechanoreceptors recorded percutaneously in awake human subjects. Exp Neurol 21:270-289.

Vallbo AB, Johansson RS (1978) The tactile sensory innervation of the glabrous skin of the human hand. In: The mechanism of recognition of objects by manipulation (Gordon $\mathrm{G}$, ed), pp 29-51. Oxford: Pergamon.

Whitsel BL, Roppolo JR, Werner G (1972) Cortical information processing of stimulus motion on primate skin. J Neurophysiol 35:691717. 